TRANSACTIONS OF THE

AMERICAN MATHEMATICAL SOCIETY

Volume 353, Number 4, Pages 1335-1370

S 0002-9947(00)02640-4

Article electronically published on December 15, 2000

\title{
EMBEDDED MINIMAL ENDS OF FINITE TYPE
}

\author{
LAURENT HAUSWIRTH, JOAQUÍN PÉREZ, AND PASCAL ROMON
}

\begin{abstract}
We prove that the end of a complete embedded minimal surface in $\mathbb{R}^{3}$ with infinite total curvature and finite type has an explicit Weierstrass representation that only depends on a holomorphic function that vanishes at the puncture. Reciprocally, any choice of such an analytic function gives rise to a properly embedded minimal end $E$ provided that it solves the corresponding period problem. Furthermore, if the flux along the boundary vanishes, then the end is $C^{0}$-asymptotic to a Helicoid. We apply these results to proving that any complete embedded one-ended minimal surface of finite type and infinite total curvature is asymptotic to a Helicoid, and we characterize the Helicoid as the only simply connected complete embedded minimal surface of finite type in $\mathbb{R}^{3}$.
\end{abstract}

\section{INTRODUCTION}

The property of having finite total curvature has been crucial in both reaching a rather exhaustive understanding and finding an abundance of examples of complete embedded minimal surfaces (CEMS) in three-dimensional Euclidean space $\mathbb{R}^{3}$; see for example the work of Hoffman and Karcher [2] for a good overview. If we consider CEMS with infinite total curvature, a first family that we find is the one containing the periodic minimal surfaces, which by considering quotients of $\mathbb{R}^{3}$ often become examples of finite topology and finite total curvature. But Hoffman, Karcher and Wei's 3] discovery of a surprising example with genus one in $\mathbb{R}^{3}$ having (truly) infinite total curvature opened a new field of research. In fact, in their pioneering work these authors only gave computational evidences of the embeddedness of the new surface, but very recently Hoffman and McCuan 4 have proved that, outside a compact set, the surface has no self-intersections and it lies in any regular neighborhood of a Helicoid. Indeed, they study the geometry of a complete punctured minimal disk on which the Weierstrass data $(g, \eta)$, given by the stereographically projected Gauss map $g$ and the height differential $\eta$, satisfy that both $d g / g$ and $\eta$ have double poles at the puncture without residues, besides the geometric hypothesis (fulfilled by the 'genus one Helicoid') of containing both a vertical and a horizontal ray.

Moreover, an appropriate starting point for studying CEMS of finite topology but infinite total curvature is assuming finite type, i.e., the surface $M \subset \mathbb{R}^{3}$ under study has the conformal type of a compact Riemann surface with a finite number of points

Received by the editors March 8, 1999 and, in revised form, September 29, 1999.

2000 Mathematics Subject Classification. Primary 53A10, Secondary 49Q05, 53C42.

Key words and phrases. Minimal surface, finite type, Helicoid.

The research of the second author was partially supported by a DGYCYT Grant No. PB970785. 
removed and the differentials $d g / g, \eta$ extend meromorphically to the punctures (this notion was first introduced in Rosenberg [10]; see also [8]). Under these conditions, a natural question would be controlling the asymptotic behavior of any CEMS of finite type and infinite total curvature. In this paper we prove, among other results, that the Helicoid models the geometry at infinity of any such surface.

Theorem 1. Let $M \subset \mathbb{R}^{3}$ be a complete embedded one-ended minimal surface of finite type, infinite total curvature and Weierstrass representation $(g, \eta)$. Then, $M$ is proper, has bounded Gaussian curvature, the meromorphic differentials $d g / g, \eta$ have double poles at the puncture without residue and $M$ is $C^{0}$-asymptotic to a Helicoid.

From the perspective given by this statement, the hypotheses in 4 about the order of poles of $d g / g$ and $\eta$ become, if possible, more reasonable. Theorem 1 follows by a combination of two results concerning the geometry of an isolated end of finite type, each one having its own interest. In the first one we show how embeddedness and completeness constrain the Weierstrass data of the end to an explicit form.

Theorem 2. Let $E \subset \mathbb{R}^{3}$ be a complete embedded minimal annular end with infinite total curvature, finite type and Weierstrass data $(g, \eta)$. Assume that the third coordinate of the flux of E along its boundary is zero. Then, $E$ can be conformally parametrized in a punctured disk $\{|z| \geq R\}$ centered at infinity by the Weierstrass data (up to isometries)

$$
g(z)=e^{i z+f(z)}, \quad \eta=d z, \quad|z| \geq R,
$$

where $f$ is a holomorphic function in $\{|z| \geq R\} \cup\{\infty\}$ vanishing at infinity.

With this notation, $f=0$ corresponds to the end of a helicoid. The second key ingredient to prove Theorem 1 deals with the inverse problem, answering whether a choice of the analytic data $(g, \eta)$ as in (1) gives rise to a complete embedded minimal end and besides that, whether the asymptotic behavior achieved in 4 remains true. With this in mind, we establish the equivalence to be expected between analytic and geometric properties.

Theorem 3. Let $R>0$ and $f$ be a holomorphic function in $\{|z| \geq R\} \cup\{\infty\}$ with $f(\infty)=0$. Suppose that the pair $\left(g(z)=e^{i z+f(z)}, \eta=d z\right)$ is the Weierstrass representation of a minimal immersion $X:\{|z| \geq R\} \longrightarrow \mathbb{R}^{3}$. Then, $X$ is proper, has bounded Gaussian curvature and there exists $R_{1} \geq R$ such that $X\left(\left\{|z| \geq R_{1}\right\}\right)$ is embedded. Moreover, if the flux of $X$ along its boundary is zero, then there exists a vertical Helicoid $H \subset \mathbb{R}^{3}$ such that for every $\varepsilon>0$, we can find $R_{1} \geq R$ depending on $\varepsilon$ such that $X\left(\left\{|z| \geq R_{1}\right\}\right)$ lies inside a regular neighborhood of $H$ of radius $\varepsilon$.

On the other hand, a great part of the machinery concerning minimal surfaces of finite type is devoted to proving an old conjecture that asks if the only nonplanar simply connected CEMS in $\mathbb{R}^{3}$ is the Helicoid. Partial answers to this conjecture have been given by in the works of Meeks and Rosenberg [5], Romon [9], Rosenberg [10], Rodriguez and Rosenberg [7, 8, Rosenberg and Toubiana [11] and Xavier [12]. Here we contribute to this partial knowledge with the following:

Theorem 4. The only simply connected complete embedded minimal surfaces of finite type are the plane and the Helicoid. 
This article also serves as an erratum for [9], where a certain configuration of the Weierstrass data was discarded by proving that the period problem cannot be solved; here we show that there exist choices of such complex data for which the period is killed, giving rise to the 'folded helicoidal end' studied in Section 6, but such an end is always nonembedded.

The paper is organized as follows. In Section 2 we fix the notations and state two lemmas on the behaviour of planar curves which will be used in Section 3 to analyze certain curves on a minimal end of finite type. Sections 4 and 5 are devoted to proving Theorems 2 and 3 respectively. Section 6 contains the corrections for [9] which are used in previous arguments. Finally, Section 7 includes the proof of Theorems 1 and 4 This paper is inspired by the work of the third author [9, which approaches a similar problem to the one tackled here, with the additional assumptions that the surface is proper and it intersects every horizontal plane in a single connected curve. The authors would like to thank Pascal Collin, Geraldo de Oliveira Filho, Lucio Rodriguez, Antonio Ros and Harold Rosenberg for helpful conversations on the subject.

\section{Preliminaries}

Given $R>0$, denote by $D(\infty, R) \subset \mathbb{C}$ the punctured disk centered at infinity $\left\{z=r e^{i \theta}: r \geq R\right\}$ and consider a nonflat minimal immersion $X: D(\infty, R) \longrightarrow \mathbb{R}^{3}$, parametrized globally by its Weierstrass data $(g, \eta)$ by

$$
X(z)=\frac{1}{2} \text { Real } \int^{z}\left(g^{-1}-g, i\left(g^{-1}+g\right), 2\right) \eta,
$$

where $g$ is the Gauss map of $X$ stereographically projected from the north pole of the sphere and $\eta$ is its height differential. Assume that the expressions of $g$ and $\eta$ are given by

$$
g(z)=z^{m} e^{H(z)}, \quad \eta=z^{k-1} d z, \quad z \in D(\infty, R),
$$

where $m, k$ are integers, $k \geq 1$, and $H$ is a holomorphic function on $D(\infty, R)$ that extends meromorphically to $\infty$. Thus $H$ can be written as

$$
H(z)=\sum_{\lambda=-\infty}^{n} a_{\lambda} z^{\lambda}
$$

where $n$ is an integer (which is supposed to be greater than or equal to one) and the $a_{\lambda}$ are complex numbers, $a_{n} \neq 0$. These assumptions on the Weierstrass data will become clear later on, when proving Theorem [2. For the sake of clarity, we will now simply suppose this normalization on $X$.

Note that as $n \geq 1$, the Gauss map $g$ has an essential singularity at the end and the total curvature of the surface $X(D(\infty, R))$ is infinite. Also, $g$ misses the values 0 and $\infty$ in $D(\infty, R)$, hence $X(D(\infty, R))$ meets transversally any horizontal plane in $\mathbb{R}^{3}$. It will be useful for our purposes to clearly separate the modulus and the 
argument of $g$, so we write

$$
\begin{aligned}
& g(z)=z^{m} e^{H(z)}=e^{A(z)+i B(z)}, \\
& \text { with }\left\{\begin{array}{l}
A(z)=m \log r+\sum_{\lambda=-\infty}^{n}\left|a_{\lambda}\right| r^{\lambda} \cos \left(\lambda \theta+\alpha_{\lambda}\right), \\
B(z)=m \theta+\sum_{\lambda=-\infty}^{n}\left|a_{\lambda}\right| r^{\lambda} \sin \left(\lambda \theta+\alpha_{\lambda}\right)
\end{array}\right.
\end{aligned}
$$

and $a_{\lambda}=\left|a_{\lambda}\right| e^{i \alpha_{\lambda}}$ for each $\lambda$. We will determine the geometry of the minimal end $X(D(\infty, R))$ by carrying out a careful study of certain curves on the surface, whose behavior is described by their horizontal projection on the $\left(x_{1}, x_{2}\right)$-plane (which we identify for the remainder of the paper with $\mathbb{C}$, so the origin always identifies with the point $\left.(0,0, c) \in\left\{x_{3}=c\right\}\right)$ and by their height with respect to this plane. From now on, $\mathcal{O}\left(s^{d}\right)$ will denote a function such that $s^{-d} \mathcal{O}\left(s^{d}\right)$ is bounded as $s \rightarrow \infty$.

The following lemma describes the geometry of certain planar curves in terms of the behavior of their tangent vectors.

Lemma 1. Let $\xi(s), s \in \mathbb{R}^{+}$be a planar curve with velocity vector $\frac{d \xi}{d s}=r(s) e^{i \varphi(s)}$, where $r(s), \varphi(s)$ are smooth real valued functions of the type

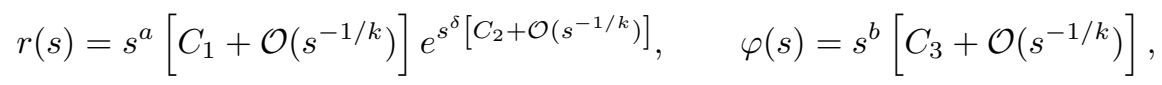

with $a, b, \delta \in \mathbb{R}, k$ is a positive integer, $b, C_{1}, C_{2}>0, C_{3} \neq 0$ (note that $\varphi(s)$ diverges to infinity) and the remainder terms $\mathcal{O}\left(s^{-1 / k}\right)$ are uniformly converging series in negative powers of $s$. Then, for s large enough:

i) If $\delta>0$ or if $\delta=0$ and $a+1>b$, then $\xi(s)$ is a proper spiral.

ii) If $\delta=0$ and $a+1=b$, then $\xi(s)$ is a spiral asymptotic to a circle.

iii) If $\delta=0$ and $a+1<b$, then $\xi(s)$ is a spiral that converges to a point.

Proof. The statement follows from Lemma 3 in the Appendix. Here we will sketch a brief idea of the proof of Lemma1, assuming the result in that Appendix. It suffices to find functions $\rho$ and $\alpha$ such that $\xi(s)=\rho(s) e^{i \alpha(s)}$ for $s$ large, $\alpha$ monotonically increasing or decreasing to infinity, and then describe the shape of $\xi$ in terms of these functions. Given a ray $l_{\alpha}$ from the origin in $\mathbb{C}$ with angle $\alpha \in[0,2 \pi)$, we consider the critical points of the function $x_{\alpha}(s)=\left\langle\xi(s), e^{i(\alpha+\pi / 2)}\right\rangle$. Lemma 3 insures that there exists an infinite countable number of such points, passing consecutively from local maxima to local minima, and that if we denote by $s_{2 n}$ (resp. $s_{2 n-1}$ ) the sequence of consecutive local maxima (resp. minima) of $x_{\alpha}$, then $x_{\alpha}\left(s_{n}\right)-x_{\alpha}\left(s_{n-1}\right)$ changes sign as $n$ increases.

In case $i$ ) we have $x_{\alpha}\left(s_{2 n}\right) \rightarrow+\infty, x_{\alpha}\left(s_{2 n-1}\right) \rightarrow-\infty$. If the ray $l_{\alpha}$ does not meet the curve tangentially, we can parametrize $\xi$ locally in polar coordinates. But a tangential intersection would produce an extremum of $x_{\alpha}$ with value zero, a contradiction. As this argument holds uniformly for every $\alpha \in[0,2 \pi), \xi$ can be globally parametrized in polar coordinates. As for any $\alpha \in[0,2 \pi)$ the sequence of local maxima (resp. minima) of the height function $x_{\alpha}$ with respect to $l_{\alpha}$ diverges to $+\infty$ (resp. to $-\infty), \xi(s)$ must be a diverging spiral.

In case ii), the local maxima and minima of $x_{\alpha}$ converge to two constants $M(\alpha), m(\alpha)$ and the distance $W$ between these limits does not depend on $\alpha$. It follows that for all $\varepsilon>0$ small, the curve $\xi$ is eventually contained in a slab parallel to $l_{\alpha}$ of width $W+\varepsilon$ but not in a slab parallel to this one of width $W-\varepsilon$, and 
this fact holds for all $\alpha \in[0,2 \pi)$. This is enough to conclude the statement in the second case.

In case iii), $\left|x_{\alpha}(s)-x_{\alpha}\left(s^{\prime}\right)\right|$ goes to zero as both $s>s^{\prime}$ tend to infinity. Hence there exists a real number $c_{\alpha}$ such that $x_{\alpha}(s)$ converges to $c_{\alpha}$ as $s \rightarrow \infty$. Changing the angle $\alpha$ we conclude that $\xi(s)$ converges to a point as $s \rightarrow \infty$. Finally, $\xi$ is a convergent spiral, because the difference $x_{\alpha}\left(s_{n}\right)-x_{\alpha}\left(s_{n-1}\right)$ changes sign as $n$ increases and this holds for all $\alpha$. This completes the proof of the lemma.

A crucial point for finding spirals in the lemma above is that $b>0$, which forces $\varphi$ to diverge. We will also need an asymptotic description of the planar curve $\xi$ in the case $b=0$. Such is the purpose of the following lemma.

Lemma 2. Let $\xi(s), s \in \mathbb{R}^{+}$be a planar curve with velocity vector $\frac{d \xi}{d s}=r(s) e^{i \varphi(s)}$, where $r(s), \varphi(s)$ are smooth real valued functions of the type

$$
r(s)=s^{a}\left[C_{1}+\mathcal{O}\left(s^{-1 / k}\right)\right] e^{s\left[C_{2}+\mathcal{O}\left(s^{-1 / k}\right)\right]}, \quad \varphi(s)=\beta+\mathcal{O}\left(s^{-1 / k}\right),
$$

with $a, \beta \in \mathbb{R}, k$ is a positive integer, $C_{1}, C_{2}>0$ and the remainder terms $\mathcal{O}\left(s^{-1 / k}\right)$ are uniformly converging series in negative powers of $s$. Then, $\xi(s)$ can be written for $s$ large enough in polar coordinates as $\xi(s)=R(s) e^{i \phi(s)}$, where $R(s)$ increases to $\infty$ and $\phi(s) \rightarrow \beta$ when $s$ goes to $\infty$.

Proof. The hypothesis on the remainder terms $\mathcal{O}\left(s^{-1 / k}\right)$ insures that when deriving such a function, the remainder term is also derived (i.e. $\frac{d}{d s}\left(\mathcal{O}\left(s^{d}\right)\right)=\mathcal{O}\left(s^{d-1}\right)$ ); moreover, this property remains true for new functions obtained by inverting or composing the previous ones. In what follows, all the remainder terms will therefore have this property.

As in the proof of Lemma1 1 we consider the function $x_{\alpha_{1}}(s)=\left\langle\xi(s), e^{i\left(\alpha_{1}+\pi / 2\right)}\right\rangle$, whose derivative is $x_{\alpha_{1}}^{\prime}(s)=r(s)$ Real $\left(e^{i \varphi(s)} \overline{e^{i\left(\alpha_{1}+\pi / 2\right)}}\right)=r(s) \sin \left(\varphi(s)-\alpha_{1}\right)$. Using the expressions of $r(s), \varphi(s)$ and choosing $\alpha_{1}$ such that $\beta-\frac{\pi}{3} \leq \alpha_{1} \leq \beta-\frac{\pi}{4}$, we have

$$
\begin{aligned}
x_{\alpha_{1}}^{\prime}(s) & =r(s)\left[\sin \left(\beta-\alpha_{1}\right)+\mathcal{O}\left(s^{-1 / k}\right)\right] \\
& =s^{a}\left[C_{1} \sin \left(\beta-\alpha_{1}\right)+\mathcal{O}\left(s^{-1 / k}\right)\right] e^{s\left[C_{2}+\mathcal{O}\left(s^{-1 / k}\right)\right]},
\end{aligned}
$$

which increases to $+\infty$ as $s \rightarrow \infty$, hence the same holds with $x_{\alpha_{1}}(s)$. Calling $\alpha_{2}=\alpha_{1}-\frac{\pi}{2}$ and reasoning in a similar way with $x_{\alpha_{2}}(s)=\left\langle\xi(s), e^{i \alpha_{1}}\right\rangle$, we obtain $x_{\alpha_{2}}^{\prime}(s)=r(s) \cos \left(\varphi(s)-\alpha_{1}\right)=r(s)\left[\cos \left(\beta-\alpha_{1}\right)+\mathcal{O}\left(s^{-1 / k}\right)\right]$, which also diverges to $+\infty$, thus $x_{\alpha_{2}}$ has the same property. It remains to prove that if we write $\xi(s)$ in polar coordinates as $R(s) e^{i \phi(s)}$, then $\phi(s) \rightarrow \beta$ as $s \rightarrow \infty$. As $\xi(s)=$ $x_{\alpha_{2}}(s) e^{i \alpha_{1}}+x_{\alpha_{1}}(s) e^{i\left(\alpha_{1}+\pi / 2\right)}$, it follows from the L'Hôpital rule that for $s$ large, $\tan \left(\phi(s)-\alpha_{1}\right)=\frac{x_{\alpha_{1}}(s)}{x_{\alpha_{2}}(s)} \sim \frac{x_{\alpha_{1}}^{\prime}(s)}{x_{\alpha_{2}}^{\prime}(s)}=\tan \left(\beta-\alpha_{1}\right)+\mathcal{O}\left(s^{-1 / k}\right)$. As both $x_{\alpha_{1}}(s), x_{\alpha_{2}}(s)$ are positive for $s$ large, we deduce that $\phi(s) \rightarrow \beta$, as desired.

\section{SPeCial CURVES}

Consider a minimal immersion $X=\left(x_{1}, x_{2}, x_{3}\right): D(\infty, R) \longrightarrow \mathbb{R}^{3}$ with Weierstrass data $(g, \eta)$ normalized as in Section 2, The horizontal projection of $X$ can 
be given in terms of $(g, \eta)$ as

$$
\left(x_{1}+i x_{2}\right)(z)=\frac{1}{2}\left(\overline{\int^{z} \frac{1}{g} \eta}-\int^{z} g \eta\right), \quad z \in D(\infty, R) .
$$

Let $\gamma \subset D(\infty, R)$ be a smooth path, with parameter $s$. The velocity vector of the horizontal projection of $X(\gamma)$ is

$$
\begin{aligned}
\frac{d}{d s}\left(\left(x_{1}+i x_{2}\right)(\gamma)\right) & =\frac{1}{2}\left(\overline{\frac{1}{g} \gamma^{k-1} \frac{d \gamma}{d s}}-g \gamma^{k-1} \frac{d \gamma}{d s}\right)=\frac{1}{2 k}\left(\overline{\frac{1}{g} \frac{d\left(\gamma^{k}\right)}{d s}}-g \frac{d\left(\gamma^{k}\right)}{d s}\right) \\
& =\frac{1}{2 k}\left(e^{-A} \frac{d\left(\bar{\gamma}^{k}\right)}{d s}-e^{A} \frac{d\left(\gamma^{k}\right)}{d s}\right) e^{i B} .
\end{aligned}
$$

Horizontal level curves. We now deal with the shape of the level curves $x_{3}^{-1}(c)$ of the height function, $c \in \mathbb{R}$. As $x_{3}(z)=\frac{1}{k} \operatorname{Real}\left(z^{k}\right)=\frac{1}{k} r^{k} \cos (k \theta)$, the horizontal section at height zero is parametrized by the $2 k$ rays $\left\{z=r e^{i \theta_{l}}: r \geq R\right\} \subset$ $D(\infty, R)$, where $\theta_{l}=l \frac{\pi}{k}-\frac{\pi}{2 k}, l=1, \ldots, 2 k$. Using the parameter $s=r^{k}$, each one of these rays is written as $\gamma_{0, l}(s)=s^{1 / k} e^{i \theta_{l}}$, hence $\gamma_{0, l}^{k}(s)= \pm i s$, where + holds if $l$ is odd and - if $l$ is even. Now equation (4) gives

$$
\frac{d}{d s}\left(\left(x_{1}+i x_{2}\right)\left(\gamma_{0, l}\right)\right)= \pm \frac{1}{k} \cosh A\left(\gamma_{0, l}(s)\right) e^{i\left(B\left(\gamma_{0, l}(s)\right)-\frac{\pi}{2}\right)},
$$

an expression that we will study later on in certain particular cases. The $2 k$ rays $\gamma_{0, l}$ divide $D(\infty, R)$ in $2 k$ sectors $S_{1}, \ldots, S_{2 k}$, so that $x_{3}$ has constant sign in each sector and changes sign when crossing cyclicly consecutive sectors. We will fix our notation so that

$$
S_{1}=D(\infty, R) \cap \arg ^{-1}(] \theta_{2 k}, \theta_{1}[), \ldots, S_{2 k}=D(\infty, R) \cap \arg ^{-1}(] \theta_{2 k-1}, \theta_{2 k}[) .
$$

Thus, $S_{1}$ is always bisected by the positive real halfline, $x_{3}$ is positive on the sectors whose subindex is odd and negative on the sectors with even subindex.

Given $c>0$ large enough, the level set $x_{3}^{-1}(c) \cap S_{l}, l=1, \ldots, 2 k$ ( $l$ odd $)$, consists of a connected arc $\gamma_{c, l}$ that can be parametrized as

$$
\gamma_{c, l}(\theta)=\left(\frac{k c}{\cos k \theta}\right)^{1 / k} e^{i \theta}, \quad \theta \in\left(\theta_{l-1}, \theta_{l}\right)
$$

thus $\gamma_{c, l}$ diverges to $\infty$ as $\theta$ goes to each extrema of $\left(\theta_{l-1}, \theta_{l}\right)$. For arbitrary $c>0$, the level set $x_{3}^{-1}(c) \cap S_{l}$ is connected if and only if $R^{k} \leq k c$ and in this case, the above description works; when $0<c<\frac{R^{k}}{k}, x_{3}^{-1}(c) \cap S_{l}$ consists of two connected components parametrized by the same expression as above, but with $\theta$ running in two disjoint subintervals of the type $\left(\theta_{l-1}, \bar{\theta}_{1}\right],\left[\bar{\theta}_{2}, \theta_{l}\right)$, where $\bar{\theta}_{1}<\bar{\theta}_{2}$ are the two solutions in $\left(\theta_{l-1}, \theta_{l}\right)$ of the equation $R^{k}=\frac{k c}{\cos k \theta}$. Moreover, the family $\left\{\gamma_{c, l}\right\}_{c>0}$ foliates the sector $S_{l}$. The level curves in $x_{3}^{-1}(c)$ for $c<0$ are analogous to the ones in the case $c>0$, but they are contained in the sectors with even subindex.

We are now interested in the geometry of the horizontal sections at height $c \neq 0$, which is given by one of their horizontal projections. We will only deal with the case $c>0$, leaving to the reader the details when $c<0$. Hence, take an odd integer $l \in\{1, \ldots, 2 k\}$ and consider the level curve $\gamma_{c, l}:\left(\theta_{l-1}, \theta_{l}\right) \rightarrow S_{l}$ (we will study the asymptotic geometry of $\gamma_{c, l}$ as $\theta$ tends to one of the extrema $\theta_{l}$ or $\theta_{l-1}$ of its interval of definition; for the sake of simplicity, we have denoted such interval as 
if $x_{3}^{-1}(c) \cap S_{l}$ were connected, but the same development works for intermediate positive heights). Thus, $\gamma_{c, l}^{k}(\theta)=\frac{k c}{\cos k \theta} e^{i k \theta}$ and $\frac{d\left(\gamma_{c, l}^{k}\right)}{d \theta}=\frac{k^{2} c i}{(\cos k \theta)^{2}}$, so (44) gives

$$
\frac{d}{d \theta}\left(\left(x_{1}+i x_{2}\right)\left(\gamma_{c, l}\right)\right)=\frac{k c}{(\cos k \theta)^{2}} \cosh A\left(\gamma_{c, l}(\theta)\right) e^{i\left(B\left(\gamma_{c, l}(\theta)\right)-\frac{\pi}{2}\right)} .
$$

For $\theta$ close to $\theta_{l}$ or to $\theta_{l-1}$, we rewrite $\gamma_{c, l}$ as a function of the new parameter $s=\frac{1}{\cos k \theta} \in\left(s_{1},+\infty\right)$, thus $\frac{d \theta}{d s}= \pm\left(k s \sqrt{s^{2}-1}\right)^{-1}$ (here + holds for $\theta$ close to $\theta_{l}$ and - for $\theta$ near $\left.\theta_{l-1}\right)$. As $\frac{d \xi}{d s}=\frac{d \xi}{d \theta} \frac{d \theta}{d s}$ for $\xi=\left(x_{1}+i x_{2}\right)\left(\gamma_{c, l}\right)$, (6) becomes

$$
\frac{d}{d s}\left(\left(x_{1}+i x_{2}\right)\left(\gamma_{c, l}\right)\right)= \pm \frac{c s}{\sqrt{s^{2}-1}} \cosh A\left(\gamma_{c, l}(s)\right) e^{i\left(B\left(\gamma_{c, l}(s)\right)-\frac{\pi}{2}\right)},
$$

with the same convention of signs as before. In order to know which is the asymptotic behavior of $\left(x_{1}+i x_{2}\right)\left(\gamma_{c, l}\right)$ and according to Lemmas 1 and 2 we need the growth orders of the modulus and argument of the last expression. First note that $\frac{s}{\sqrt{s^{2}-1}}=1+\mathcal{O}\left(s^{-2}\right)$. On the other hand, evaluating (3) on the level curve $\gamma_{c, l}(s)$ we obtain

$$
\begin{gathered}
A\left(\gamma_{c, l}(s)\right)=\frac{m}{k} \log (k c s)+\sum_{\lambda=-\infty}^{n}\left|a_{\lambda}\right|(k c s)^{\lambda / k} \cos \left(\lambda \theta(s)+\alpha_{\lambda}\right), \\
B\left(\gamma_{c, l}(s)\right)=m \theta(s)+\sum_{\lambda=-\infty}^{n}\left|a_{\lambda}\right|(k c s)^{\lambda / k} \sin \left(\lambda \theta(s)+\alpha_{\lambda}\right),
\end{gathered}
$$

and therefore, the growth orders of $A\left(\gamma_{c, l}(s)\right), B\left(\gamma_{c, l}(s)\right)$ depend on the coefficients of the terms $s^{\lambda / k}$ in the expansion of $H$. We will study these orders when $\theta \rightarrow \theta_{l}$, and a similar situation will hold for $\theta \rightarrow \theta_{l-1}$. Note that derivation in the equation $\cos k \theta(s)=s^{-1}$ gives $\theta^{\prime}(s)=\frac{1}{k \sin k \theta(s)} s^{-2}$, so the $\lambda$-th term in the expansion of $\operatorname{Real} H\left(\gamma_{c, l}(s)\right)$ is

$$
\left|a_{\lambda}\right|(k c s)^{\lambda / k}\left[\cos \left(\lambda \theta_{l}+\alpha_{\lambda}\right)-\frac{\lambda \sin \left(\lambda \theta_{l}+\alpha_{\lambda}\right)}{k \sin k \theta_{l}} s^{-1}+\mathcal{O}\left(s^{-2}\right)\right] .
$$

Therefore, if $\left|a_{\lambda}\right| \cos \left(\lambda \theta_{l}+\alpha_{\lambda}\right) \neq 0$, then the $\lambda$-th term of Real $H\left(\gamma_{c, l}(s)\right)$ is of the type $s^{\lambda / k}\left[C c^{\lambda / k}+\mathcal{O}\left(s^{-1}\right)\right]$ for a certain $C \in \mathbb{R}-\{0\}$, while if $a_{\lambda} \neq 0$ but $\cos \left(\lambda \theta_{l}+\alpha_{\lambda}\right)=0$ the $\lambda$-th term of $\operatorname{Real} H\left(\gamma_{c, l}(s)\right)$ is of the type $s^{\frac{\lambda}{k}-1}\left[C c^{\lambda / k}+\mathcal{O}\left(s^{-1}\right)\right]$, and this holds for all $c>0$. These alternatives suggest that we define $\hat{\lambda}$ as the largest $\lambda \in\{1, \ldots, n\}$ such that $\left|a_{\lambda}\right| \cos \left(\lambda \theta_{l}+\alpha_{\lambda}\right) \neq 0$ (recall that $a_{n} \neq 0$ ). If no such $\lambda$ exists, we define $\widehat{\lambda}$ as zero. Note that $\widehat{\lambda}$ does not depend on the height $c$, but only on the limit angle $\theta_{l}$.

Claim 1. The growth of RealH( $\left.\gamma_{c, l}(s)\right)$ for $s$ large is as follows:

i) If $\frac{\widehat{\lambda}}{k} \neq \frac{n}{k}-1$, then for all $c \neq 0$ we have $\operatorname{Real} H\left(\gamma_{c, l}(s)\right)=s^{\delta}\left[\widetilde{C}+\mathcal{O}\left(s^{-1 / k}\right)\right]$, where $\delta=\max \left(\frac{\widehat{\lambda}}{k}, \frac{n}{k}-1\right) \geq 0$.

ii) If $\frac{\widehat{\lambda}}{k}=\frac{n}{k}-1$, then for $|c|$ large we have $\operatorname{Real} H\left(\gamma_{c, l}(s)\right)=s^{\frac{n}{k}-1}\left[\widetilde{C}+\mathcal{O}\left(s^{-1 / k}\right)\right]$. (Here $\widetilde{C} \in \mathbb{R}-\{0\}$ depends on $c$ ).

Proof of Claim 1. First of all, we make a remark concerning a technical property to be used from now on in the paper. The remainder terms $\mathcal{O}\left(s^{-1 / k}\right)$ quoted in Claim 1 are actually series in negative powers of $s$ converging uniformly in 
$\left\{s>s_{0}\right\}$ (we originally took real or imaginary parts of a Laurent series, obtaining real analytic data $A$ and $B$; this shows that after changing the parameter to $s$, all these expressions become convergent power series in $s^{-1 / k}$ ); see also the beginning of the proof of Lemma 2 .

We now prove Claim 1. Suppose that $\hat{\lambda}=n$. Then, the $n$-th term of Real $H\left(\gamma_{c, l}(s)\right)$ is $s^{n / k}\left[C+\mathcal{O}\left(s^{-1}\right)\right](C$ denotes a nonzero real number depending on $c$ ) while those terms with $\lambda \leq n-1$ are as $\mathcal{O}\left(s^{\frac{n-1}{k}}\right)$, so the claim holds in the case $\widehat{\lambda}=n$. Now assume $\widehat{\lambda}<n$, which implies that the terms of Real $H\left(\gamma_{c, l}(s)\right)$ with largest order growth are the $n$-th term, which grows as $s^{\frac{n}{k}-1}\left[C+\mathcal{O}\left(s^{-1}\right)\right]$, and the $\widehat{\lambda}$-term, whose growth is as $s^{\widehat{\lambda} / k}\left[C+\mathcal{O}\left(s^{-1}\right)\right]$. Now the expression of Real $H\left(\gamma_{c, l}(s)\right)$ for arbitrary $c \neq 0$ can be easily deduced, provided that $\frac{\widehat{\lambda}}{k} \neq \frac{n}{k}-1$. In the case $\frac{\widehat{\lambda}}{k}=\frac{n}{k}-1$ we should show that for $|c|$ large enough, the two above terms do not cancel. But this is true because the coefficient $C$ in the $n$-th term grows in $c$ as $c^{n / k}$, while the one in the $\widehat{\lambda}$-th term grows in $c$ as $c^{\widehat{\lambda} / k}$, hence both $C$-coefficients are distinct for $|c|$ large, thereby finishing the proof of Claim 1.

Using that

$$
\cosh A\left(\gamma_{c, l}(s)\right)=\frac{1}{2}\left[(k c s)^{\frac{m}{k}} e^{\operatorname{Real}\left(H\left(\gamma_{c, l}(s)\right)\right.}+(k c s)^{-\frac{m}{k}} e^{-\operatorname{Real}\left(H\left(\gamma_{c, l}(s)\right)\right.}\right],
$$

a direct computation that only discusses the signs of $\widetilde{C}$ and of $m$ gives that for $s$ large, the growth of $\cosh A\left(\gamma_{c, l}\right)$ as $\theta \rightarrow \theta_{l}$ is of the following type:

- If $\frac{\widehat{\lambda}}{k} \neq \frac{n}{k}-1$, then for arbitrary $c \neq 0$ is

$$
\cosh A\left(\gamma_{c, l}(s)\right)=s^{a}\left[C_{1}+\mathcal{O}\left(s^{-1 / k}\right)\right] e^{s^{\delta}\left[C_{2}+\mathcal{O}\left(s^{-1 / k}\right)\right]},
$$

where $C_{1}, C_{2}>0$, and $a= \pm \frac{m}{k}$.

- If $\frac{\widehat{\lambda}}{k}=\frac{n}{k}-1$, then for $|c|$ large the same expression (10) holds with $\delta=\frac{n}{k}-1$. This description together with equality (7) allows us to write the modulus of $\frac{d}{d s}\left(\left(x_{1}+i x_{2}\right)\left(\gamma_{c, l}\right)\right)$ in a suitable form for using Lemma 1 see the expression of $r(s)$ in that lemma.

Following the same ideas as before, we define $\widehat{\lambda}^{\prime}$ as the largest $\lambda \in\{1, \ldots, n\}$ such that $\left|a_{\lambda}\right| \sin \left(\lambda \theta_{l}+\alpha_{\lambda}\right) \neq 0\left(\widehat{\lambda}^{\prime}=0\right.$ if no such $\lambda$ exists), and with similar arguments as in Claim 1 the following can be proved

Claim 2. The growth of $\operatorname{Imag} H\left(\gamma_{c, l}(s)\right)$ for $s$ large is:

i) If $\frac{\widehat{\lambda}^{\prime}}{k} \neq \frac{n}{k}-1$, then for all $c \neq 0$ it holds that

$$
\operatorname{Imag} H\left(\gamma_{c, l}(s)\right)=s^{b}\left[\widehat{C}+\mathcal{O}\left(s^{-1 / k}\right)\right], \quad \text { where } b=\max \left(\frac{\widehat{\lambda}^{\prime}}{k}, \frac{n}{k}-1\right) \geq 0 .
$$

ii) If $\frac{\widehat{\lambda}^{\prime}}{k}=\frac{n}{k}-1$, then for $|c|$ large we have

$$
\operatorname{Imag} H\left(\gamma_{c, l}(s)\right)=s^{\frac{n}{k}-1}\left[\widehat{C}+\mathcal{O}\left(s^{-1 / k}\right)\right] .
$$

(Here $\widehat{C} \in \mathbb{R}-\{0\}$ depends on $c$.)

From (7) and this description one has that the argument of $\frac{d}{d s}\left(\left(x_{1}+i x_{2}\right)\left(\gamma_{c, l}\right)\right)$ looks like the function $\varphi(s)$ in Lemma 1 provided that $b>0$.

Next we state a criterion of self-intersection, to be used when proving Theorem 2 
Proposition 1. Let $X: D(\infty, R) \longrightarrow \mathbb{R}^{3}$ be a minimal immersion with the normalization on its Weierstrass data given in Section 2 . Assume that one of the following conditions holds:

i) $n$ is not a multiple of $k$.

ii) $n=k$ and $a_{k}^{2} \notin \mathbb{R}$.

iii) $n=k, a_{k} \in i \mathbb{R}$ and there exists $\lambda \in\{1, \ldots, k-1\}$ such that $a_{\lambda} \neq 0$.

iv) $n=k$ odd, $a_{k} \in \mathbb{R}$ and $\left(m ; a_{1}, \ldots a_{k-1}\right) \neq(0 ; 0, \ldots, 0)$.

v) $n=k$ even, $a_{k} \in \mathbb{R}$ and

$$
\begin{aligned}
& \left(m ; a_{1}, \ldots, a_{\frac{k}{2}-1}, a_{\frac{k}{2}}, a_{\frac{k}{2}+1}, \ldots, a_{k-1}\right) \\
& \quad \neq\left(0 ; 0, \ldots, 0,\left|a_{\frac{k}{2}}\right| e^{i\left(\frac{\pi}{4}+j \frac{\pi}{2}\right)}, 0, \ldots, 0\right), \quad j \in\{1, \ldots, 4\} .
\end{aligned}
$$

Then, there exists $c>0$ such that $X\left(x_{3}^{-1}(c)\right)$ contains two proper spirals winding in opposite directions. In particular, these spirals meet each other infinitely often and $X(D(\infty, R))$ cannot be embedded.

Proof. i) Suppose first that $n$ is not a multiple of $k$. By Lemma $4 i$ ) with $\lambda=n$ and $\alpha=\alpha_{n}$ (see Appendix), there exist $l_{1}, l_{2} \in\{1, \ldots, 2 k\}$ such that $\sin \left(n \theta_{l_{1}}+\alpha_{n}\right) \sin \left(n \theta_{l_{2}}+\alpha_{n}\right)<0$ and $l_{1}-l_{2}$ is even. Fix $c \neq 0$ and note that when studying $\operatorname{Imag} H\left(\gamma_{c, l}\right)$ at the branches of $\gamma_{c, l_{1}}, \gamma_{c, l_{2}}$ with $\theta \rightarrow \theta_{l_{1}}$ and $\theta \rightarrow \theta_{l_{2}}$ respectively, one obtains $\widehat{\lambda}^{\prime}=n$ in both cases. Moreover, the coefficient $\widehat{C}$ of the term $s^{n / k}$ in $\operatorname{Imag} H\left(\gamma_{c, l}(s)\right)$ is $\left|a_{n}\right|(k c)^{n / k} \sin \left(n \theta_{l_{i}}+\alpha_{n}\right)$, which has opposite signs at $\theta_{l_{1}}$ and at $\theta_{l_{2}}$. This implies that for $c \neq 0$ fixed, $B\left(\gamma_{c, l}\right)$ diverges to $+\infty$ when $\theta \rightarrow \theta_{l_{1}}$ on the curve $\gamma_{c, l_{1}}$ while it diverges to $-\infty$ when $\theta \rightarrow \theta_{l_{2}}$ on $\gamma_{c, l_{2}}$ (or vice versa), and both curves have images by $X$ contained in the same horizontal plane at height $c$. As $b>0$ in both cases, Lemma 1 applies to the branch of $\left(x_{1}+i x_{2}\right)\left(\gamma_{c, l_{1}}\right)$ where $\theta$ goes to $\theta_{l_{1}}$ and to the branch of $\left(x_{1}+i x_{2}\right)\left(\gamma_{c, l_{2}}\right)$ where $\theta$ goes to $\theta_{l_{2}}$, so each one of these planar branches are spirals. Moreover, if $n>k$, then Claim 1 i) insures that $\delta \geq \frac{n}{k}-1>0$ at both branches, and Lemma 1 i) implies that both spirals are divergent. As they have opposite winding directions, they will cut each other infinitely many times. If $n<k$, then we discuss two possibilities: first suppose that none of $\widehat{\lambda}\left(\theta_{l_{1}}\right), \widehat{\lambda}\left(\theta_{l_{2}}\right)$ are zero. In this case, Claim $\left.1 i\right)$ implies that both $\delta\left(\theta_{l_{1}}\right), \delta\left(\theta_{l_{2}}\right)$ are strictly positive and we conclude as before. Otherwise, at least one of the $\widehat{\lambda}\left(\theta_{l_{i}}\right)$ (say $i=1$ ) vanishes, and it suffices to check that in this case we have $\delta\left(\theta_{l_{1}}\right)=0$ and $a+1>b\left(\theta_{l_{1}}\right)$ so we can use again Lemma 1 $\left.i\right)$. As $n<k$ and $\widehat{\lambda}\left(\theta_{l_{1}}\right)=0$, then Claim $1 i$ ) assures that $\delta\left(\theta_{l_{1}}\right)=0$, thus Real $(H)$ remains bounded along $\gamma_{c, l_{1}}$ as $\theta \rightarrow \theta_{l_{1}}$ and the exponent $a$ in equation (10) becomes $\frac{|m|}{k}$. In particular, $a+1 \geq 1>\frac{n}{k}=b\left(\theta_{l_{1}}\right)$, where this last equality follows from $\widehat{\lambda}^{\prime}\left(\theta_{l_{1}}\right)=n$. This proves $i$ ).

ii) Now assume that $n=k$ and $a_{k}^{2} \notin \mathbb{R}$. Given $l \in\{1, \ldots, 2 k\}$ fixed, note that neither $\sin \left(k \theta_{l}+\alpha_{k}\right)$ nor $\cos \left(k \theta_{l}+\alpha_{k}\right)$ vanish. As $\theta_{l}-\theta_{l-1}=\frac{\pi}{k}$, it follows that $\sin \left(k \theta_{l}+\alpha_{k}\right) \sin \left(k \theta_{l-1}+\alpha_{k}\right)<0$ and $\cos \left(k \theta_{l-1}+\alpha_{k}\right) \neq 0$. In particular, $\widehat{\lambda}=\widehat{\lambda}^{\prime}=k$ (thus we are in case $i$ ) of Claim 1 with $\delta=1$; analogously, $b=1$ ) at both extrema $\theta_{l}$ and $\theta_{l-1}$ on the curve $\gamma_{c, l}$ for $c \neq 0$ fixed, and the coefficients $\widehat{C}$ in Claim 2 have opposite signs. Now we finish as in case $i$ ), and the second case is also proved.

iii) Suppose now that $n=k, a_{k} \in i \mathbb{R}$ and there exists $\lambda \in\{1, \ldots, k-1\}$ such that $a_{\lambda} \neq 0$. Reasoning as in ii) we have $\cos \left(k \theta_{l}+\alpha_{k}\right) \neq 0$ and $\sin \left(k \theta_{l}+\alpha_{k}\right)=0$, which imply that $\widehat{\lambda}=k$ (i.e. we are again in case $i$ ) of Claim 1 with $\delta=1$ ) and 
$\widehat{\lambda}^{\prime}<k$ for each $l$. Consider the largest $\lambda \in\{1, \ldots, k-1\}$ such that $a_{\lambda} \neq 0$. Using Lemma $4 i$ ) with these $k, \lambda$ we deduce that there exist $l_{1}, l_{2} \in\{1, \ldots, 2 k\}$ with $\sin \left(\lambda \theta_{l_{1}}+\alpha_{\lambda}\right) \sin \left(\lambda \theta_{l_{2}}+\alpha_{\lambda}\right)<0$ and $l_{1}-l_{2}$ even. Thus, $\widehat{\lambda}^{\prime}\left(\theta_{l_{1}}\right)=\widehat{\lambda}^{\prime}\left(\theta_{l_{2}}\right)=\lambda>0$ and Claim 2 i) insures that $b=\frac{\lambda}{k}>0$ in both cases, again with the coefficients $\widehat{C}$ of opposite signs, thereby proving iii).

The cases iv) and $v$ ) can be treated as a single one; for simplicity, we just write $n=k, a_{k} \in \mathbb{R}$ and

$$
\begin{aligned}
& \left(m ; a_{1}, \ldots, a_{\frac{k}{2}-1}, a_{\frac{k}{2}}, a_{\frac{k}{2}+1}, \ldots, a_{k-1}\right) \\
& \quad \neq\left(0 ; 0, \ldots, 0,\left|a_{\frac{k}{2}}\right| e^{i\left(\frac{\pi}{4}+j \frac{\pi}{2}\right)}, 0, \ldots, 0\right), \quad j \in\{1, \ldots, 4\},
\end{aligned}
$$

understanding that when $k$ is odd there is no $a_{\frac{k}{2}}$ to consider. Then, $\cos \left(k \theta_{l}+\alpha_{k}\right)=0$ and $\sin \left(k \theta_{l}+\alpha_{k}\right) \neq 0$, which imply $\hat{\lambda}<k$ and $\widehat{\lambda}^{\prime}=k$. In particular, we are again in case $i$ ) of Claim 2 with $b=1$ at each $\theta_{l}, l=1, \ldots, 2 k$, for a level curve at any fixed height $c \neq 0$. Similarly, as in case ii) of this proposition, we have that $\sin \left(k \theta_{l}+\alpha_{k}\right) \sin \left(k \theta_{l-1}+\alpha_{k}\right)<0$ thus the coefficient $\widehat{C}$ in $\operatorname{Imag} H\left(\gamma_{c, l}(s)\right)$ has opposite signs at both extrema $\theta_{l}, \theta_{l-1}$. If $m \neq 0$, then (8) insures that $A \circ \gamma_{c, l}$ is not bounded as $\theta \rightarrow \theta_{l}$ or $\theta \rightarrow \theta_{l-1}$, hence the same holds with $\cosh A\left(\gamma_{c, l}(s)\right)$. Take $|c|$ large enough, so we can insure that $\cosh A$ is as in (10) (note that $\frac{\widehat{\lambda}}{k}$ could be $0=\frac{n}{k}-1$ ). As a consequence, either $\delta>0$ or $a>0$, and both situations imply that we are in case $i$ ) of Lemma 1 at both extrema of $\gamma_{c, l}$, so we can find a self-intersection as in former cases. Finally, when $m=0$ then there exists $\lambda \in\{1, \ldots, k-1\}$ such that $a_{\lambda} \neq 0$ and $\left(\lambda, \alpha_{\lambda}\right) \neq\left(\frac{k}{2}, \frac{\pi}{4}+j \frac{\pi}{2}\right)$ with $j=1, \ldots, 4$, and Lemma 4 ii) insures that there exists $l_{0} \in\{1, \ldots, 2 k\}$ such that $\cos \left(\lambda \theta_{l_{0}}+\alpha_{\lambda}\right) \neq 0$ and $\cos \left(\lambda \theta_{l_{0}-1}+\alpha_{\lambda}\right) \neq 0$, which implies that both $\widehat{\lambda}\left(\theta_{l_{0}}\right)$ and $\widehat{\lambda}\left(\theta_{l_{0}-1}\right)$ are positive, thus both branches of $X\left(\gamma_{c, l_{0}}\right)$ as $\theta \rightarrow \theta_{l_{0}}$ and as $\theta \rightarrow \theta_{l_{0}-1}$ are in case $\left.i\right)$ of Claim 1 for all height $c \neq 0$ with $\delta\left(\theta_{l_{0}}\right), \delta\left(\theta_{l_{0}-1}\right)>0$ and with coefficients $\widehat{C}$ in $\operatorname{Imag} H\left(\gamma_{c, l_{0}}(s)\right)$ of opposite signs, and we finish as before.

Remark 1. Following the notation above, if $n=k$ and $a_{k} \in \mathbb{R}$ one possibility remains which is not covered by Proposition 11 namely, when there exists $j \in$ $\{1, \ldots, 4\}$ such that

$$
\left(m ; a_{1}, \ldots, a_{\frac{k}{2}-1}, a_{\frac{k}{2}}, a_{\frac{k}{2}+1}, \ldots, a_{k-1}\right)=\left(0 ; 0, \ldots, 0,\left|a_{\frac{k}{2}}\right| e^{i\left(\frac{\pi}{4}+j \frac{\pi}{2}\right)}, 0, \ldots, 0\right)
$$

(as before, when $k$ is odd we agree to use this notation but no $a_{\frac{k}{2}}$ is considered). We affirm that in this setting, the immersion is not proper. This holds because there exists $l \in\{1, \ldots, 2 k\}$ such that $\widehat{\lambda}\left(\theta_{l}\right)=0$ and $\widehat{\lambda}^{\prime}\left(\theta_{l}\right)=k$ (when $k$ is odd, any choice of $l$ works; when $k$ is even, choose $l$ such that $\frac{k}{2} \theta_{l}+\alpha_{\frac{k}{2}} \equiv \frac{\pi}{2} \bmod \pi$, which is possible because $\alpha_{\frac{k}{2}}=\frac{\pi}{4}+j \frac{\pi}{2}$ ), thus we are in case ii) of Claim 1. In particular, Real $H\left(\gamma_{c, l}(s)\right)$ remains bounded for $|c|$ large and as $m=0$, the same holds with $\cosh A\left(\gamma_{c, l}\right)$ as $\theta \rightarrow \theta_{l}$. With the notation of Lemma 1, this means that the velocity vector of $\frac{d}{d s}\left(x_{1}+i x_{2}\right)\left(\gamma_{c, l}(s)\right)$ for $|c|$ large has $\delta=0$ and $a+1=1=b$, thus the branch of $X\left(\gamma_{c, l}\right)$ when $\theta \rightarrow \theta_{l}$ lies in case ii) of Lemma 1 ,

Our next goal is to describe the case $n=k, a_{k} \in i \mathbb{R}$ and $a_{1}=\ldots=a_{k-1}=0$, which is not tackled neither by Proposition 1 nor by Remark 1. Under these conditions we can no longer apply Lemma 1, because our hypotheses force $B\left(\gamma_{c, l}\right)$ to remain bounded as $\theta \rightarrow \theta_{l}, \theta_{l-1}$ for all $c \neq 0$ and all $l$, and now we use (77) to 
deduce that $b=0$ in the notation of Lemma 11. Nevertheless, we can still give a geometric description of the curves $X\left(\gamma_{c, l}\right)$ for arbitrary $c$ :

Proposition 2. Let $X: D(\infty, R) \longrightarrow \mathbb{R}^{3}$ be a minimal immersion with the normalization of Section $\left[\right.$ Assume that $n=k, a_{k} \in i \mathbb{R}$ and $a_{1}=\ldots=a_{k-1}=0$. Then, for all $c \in \mathbb{R}$ each horizontal level curve $X\left(\gamma_{c, l}\right)$ has two divergent branches whose arguments converge to the arguments of two halfines starting from the origin in $\left\{x_{3}=c\right\}$. Moreover, the angle between these halflines remains constant but the halflines rotate at constant speed around the $x_{3}$ axis as $c$ increases.

Proof. We first analyze the height zero. Using the parametrization $\gamma_{0, l}(s)=$ $s^{1 / k} e^{i \theta_{l}}$, the expansions in (3) become

$$
\begin{aligned}
& A\left(\gamma_{0, l}(s)\right)=\frac{m}{k} \log s+\mathcal{O}\left(s^{-1 / k}\right)+\operatorname{Real}\left(a_{0}\right) \pm\left|a_{k}\right| s, \\
& B\left(\gamma_{0, l}(s)\right)=m \theta_{l}+\mathcal{O}\left(s^{-1 / k}\right)+\operatorname{Imag}\left(a_{0}\right),
\end{aligned}
$$

thus equation (5) says that the modulus and the argument of $\frac{d}{d s}\left(\left(x_{1}+i x_{2}\right)\left(\gamma_{0, l}\right)\right)$ as $s$ becomes large can be written as in Lemma 2 Concerning the height $c \neq 0$, equations (8) and (9) are now

$$
\begin{aligned}
& A\left(\gamma_{c, l}(s)\right)=\frac{m}{k} \log (k c s)+\mathcal{O}\left(s^{-1 / k}\right)+\operatorname{Real}\left(a_{0}\right) \pm\left|a_{k}\right| k c s, \\
& B\left(\gamma_{c, l}(s)\right)=\beta+\mathcal{O}\left(s^{-1 / k}\right),
\end{aligned}
$$

for a certain contant $\beta \in \mathbb{R}$, and thus (7) assures that the asymptotic behavior of both branches of $X\left(\gamma_{c, l}\right)$ is also described by Lemma 2 From now on, both cases $c=0$ and $c \neq 0$ can be treated as a single one. Applying Lemma 2 to each branch of $X\left(\gamma_{c, l}\right)$ (for $c=0$ we understand that the two branches come from two consecutive rays $\left.\gamma_{0, l-1}, \gamma_{0, l}\right)$, we deduce that these horizontal curves have arguments asymptotic to two fixed angles, which determine two halflines $L_{l}$ (when the angle $\theta$ in the parameter domain tends to $\theta_{l}$ ) and $L_{l-1}$ (when $\theta \rightarrow \theta_{l-1}$ ), both contained in $\left\{x_{3}=c\right\}$. Thus, in order to prove our proposition we only need to compute the limit angles of the argument in (5) and in (6), i.e. $B\left(\theta_{l}\right)-\frac{\pi}{2}, B\left(\theta_{l-1}\right)-\frac{\pi}{2}$ (in the case $c=0$ this notation must be understood with its obvious meaning). From the expression of $B\left(\gamma_{0, l}(s)\right)$ above we directly have that at height zero is $B\left(\theta_{l}\right)=m \theta_{l}+\operatorname{Imag}\left(a_{0}\right)$ while $B\left(\theta_{l-1}\right)=m \theta_{l-1}+\operatorname{Imag}\left(a_{0}\right)$. As $H(z)=a_{k} z^{k}+a_{0}+f(z)$ where $f$ is a holomorphic function in a neighborhood of $\infty$ that vanishes at this point, for nonzero height $c$ we have $B\left(\gamma_{c, l}(\theta)\right)=m \theta+\left|a_{k}\right| k c \frac{\sin \left(k \theta+\alpha_{k}\right)}{\cos k \theta}+\operatorname{Imag}\left[a_{0}+f\left(\gamma_{c, l}(\theta)\right)\right]$. As $a_{k} \in i \mathbb{R}$, it follows that $\sin \left(k \theta_{l}+\alpha_{k}\right)=\sin \left(k \theta_{l-1}+\alpha_{k}\right)=0$, so by expanding $\frac{\sin \left(k \theta+\alpha_{k}\right)}{\cos k \theta}$ in series around $\theta_{l}, \theta_{l-1}$ we obtain

$$
B\left(\gamma_{c, l}(\theta)\right)=m \theta-\left|a_{k}\right| k c \frac{\cos \left(k \theta+\alpha_{k}\right)}{\sin k \theta}+\operatorname{Imag}\left(a_{0}\right)+\mathcal{O}\left(\theta-\theta_{\mu}\right),
$$

with $\mu=l-1, l$. As the quotient $\frac{\cos \left(k \theta+\alpha_{k}\right)}{\sin k \theta}$ has the same value $\varepsilon= \pm 1$ (that only depends on the sign of $\left.\operatorname{Imag}\left(a_{k}\right)\right)$ at $\theta=\theta_{l}$ and at $\theta=\theta_{l-1}$, we deduce that at height $c \neq 0$ is $B\left(\theta_{l}\right)=m \theta_{l}-\left|a_{k}\right| k c \varepsilon+\operatorname{Imag}\left(a_{0}\right)$ while $B\left(\theta_{l-1}\right)=B\left(\theta_{l}\right)+m\left(\theta_{l-1}-\theta_{l}\right)$. Therefore, the equality $\left|B\left(\theta_{l}\right)-B\left(\theta_{l-1}\right)\right|=|m|\left(\theta_{l}-\theta_{l-1}\right)=\frac{|m|}{k} \pi$ holds for all height $c$ and it shows that the halflines $L_{l-1}, L_{l}$ form an angle that does not depend on $c$. Moreover, the linear dependence of $B\left(\theta_{l}\right)$ with respect to $c \neq 0$ proves that these halflines rotate linearly with $c$ around the $x_{3}$ axis.

Remark 2. i) As the argument of a given branch of $X\left(\gamma_{c, l}\right)$ converges to the argument along one of the halflines $L$ in the statement of Proposition 2, the 
branch of $X\left(\gamma_{c, l}\right)$ can be expressed as a sublinear graph over $L$. It may be the case that $X\left(\gamma_{c, l}\right)$ is not asymptotic to $L$ or even that the orthogonal distance of $X\left(\gamma_{c, l}\right)$ to $L$ is unbounded, as in a logarithmic graph. One example of this situation can be found in [4], Remark 4 .

ii) From the proof above, we deduce that the limit angles of the two halflines $L_{l-1}, L_{l}$ are $B\left(\theta_{l-1}\right) \pm \pi$ and $B\left(\theta_{l}\right)$ respectively. On the other hand, when $m=0$ we obtain $B\left(\theta_{l-1}\right)=B\left(\theta_{l}\right)$, which means that the two halflines $L_{l-1}, L_{l}$ have opposite directions, thus they form a complete straightline, as in the helicoid.

iii) The 'space' that each $X\left(\gamma_{c, l}\right)$ takes in its horizontal plane can be related with the space covered by an angular sector with the same limit angles. Taking into account that we must stack $k$ sectors of the same amplitude at height $c$, embeddedness condition produces certain upper bound of this amplitude, which can be translated into an inequality between $|m|$ and $k$. As this information will not be used later on, we omit a detailed development of such inequality.

Preimages of spherical parallels and meridians. We now study the set of points on $X(D(\infty, R))$ where the Gauss map lies in a horizontal parallel of the sphere. From (2) we know that $H$ is asymptotic at $\infty$ to a polynomial of degree $n$, hence for $|z|$ large, the set $\{\operatorname{Real}(H(z))=0\} \subset D(\infty, R)$ consists of $2 n$ divergent curves asymptotic to the $2 n$ rays of an equiangular system centered at $\infty$. As $\operatorname{Real}(H(z))=r^{n}\left[\left|a_{n}\right| \cos \left(n \theta+\alpha_{n}\right)+\mathcal{O}\left(r^{-1}\right)\right]$, the argument along each one of these divergent curves converges to $\Theta_{j}=\frac{\pi-2 \alpha_{n}}{2 n}+j \frac{\pi}{n}, j=1, \ldots, 2 n$.

Given $A \in \mathbb{R}$, the condition $|g(z)|=e^{A}$ becomes

$$
m \log r+\sum_{\lambda=-\infty}^{n}\left|a_{\lambda}\right| r^{\lambda} \cos \left(\lambda \theta+\alpha_{\lambda}\right)=A
$$

or equivalently,

$$
\cos \left(n \theta+\alpha_{n}\right)=\frac{A}{\left|a_{n}\right| r^{n}}-\frac{m \log r}{\left|a_{n}\right| r^{n}}-\sum_{\lambda=-\infty}^{n-1} \frac{\left|a_{\lambda}\right| \cos \left(\lambda \theta+\alpha_{\lambda}\right)}{\left|a_{n}\right| r^{n-\lambda}} .
$$

As a consequence, $|g|^{-1}\left(e^{A}\right) \subset D(\infty, R)$ consists, for $|z|$ large, of $2 n$ divergent curves $\Gamma_{1}^{A}, \ldots, \Gamma_{2 n}^{A}$ asymptotic to the rays of angle $T_{j}, j=1, \ldots, 2 n$, where $\{\operatorname{Real}(H)=0\}$ also converges. We will use Lemma 1 again in order to describe the geometry of $X\left(\Gamma_{j}^{A}\right)$. First, parametrize $\Gamma_{j}^{A}$ by $r=|z|$, thus we can write $\Gamma_{j}^{A}(r)=r e^{i \theta_{j}^{A}(r)}$, where $\theta=\theta_{j}^{A}(r)$ is defined by equation (12). Assuming $n \geq 2$ or $(n, m)=(1,0)$, derivation in (12) gives $\theta_{j}^{A}(r)=\Theta_{j}+\mathcal{O}\left(r^{-1}\right)$, hence using the reparametrization $s=r^{k}$ we have

$$
\Gamma_{j}^{A}(s)=s^{1 / k} e^{i\left[\Theta_{j}+\mathcal{O}\left(s^{-1 / k}\right)\right]} \text { and } \frac{d\left(\left(\Gamma_{j}^{A}\right)^{k}\right)}{d s}=\left[1+\mathcal{O}\left(s^{-1 / k}\right)\right] e^{i\left[k \Theta_{j}+\mathcal{O}\left(s^{-1 / k}\right)\right]} .
$$

Now (4) implies

$$
\frac{d}{d s}\left(\left(x_{1}+i x_{2}\right)\left(\Gamma_{j}^{A}\right)\right)=\left[\frac{-1}{k} \sinh \left(A+i k \Theta_{j}\right)+\mathcal{O}\left(s^{-1 / k}\right)\right] e^{i B\left(\Gamma_{j}^{A}(s)\right)},
$$


and as

$$
\begin{aligned}
B\left(\Gamma_{j}^{A}(r)\right)= & m \theta_{j}^{A}(r)+\operatorname{Imag}(H)=m\left[\Theta_{j}+\mathcal{O}\left(r^{-1}\right)\right] \\
& +\left[\left|a_{n}\right| r^{n} \sin \left(n \theta_{j}^{A}(r)+\alpha_{n}\right)+\mathcal{O}\left(r^{n-1}\right)\right] \\
= & r^{n}\left[\left|a_{n}\right| \sin \left(n \Theta_{j}+\alpha_{n}\right)+\mathcal{O}\left(r^{-1}\right)\right],
\end{aligned}
$$

we finally obtain

$$
\frac{d}{d s}\left(\left(x_{1}+i x_{2}\right)\left(\Gamma_{j}^{A}\right)\right)=\left[\frac{-1}{k} \sinh \left(A+i k \Theta_{j}\right)+\mathcal{O}\left(s^{-1 / k}\right)\right] e^{i s^{n / k}\left[C+\mathcal{O}\left(s^{-1 / k}\right)\right]}
$$

where $C= \pm\left|a_{n}\right|$ is a nonzero real number (note that $\sin \left(n \Theta_{j}+\alpha_{n}\right)= \pm 1$ ).

On the other hand, the height of the curve $X\left(\Gamma_{j}^{A}\right)$ is given by

$$
x_{3}\left(\Gamma_{j}^{A}(r)\right)=\frac{1}{k} r^{k} \cos k \theta_{j}^{A}(r)=\frac{1}{k} r^{k} \cos \left(k \Theta_{j}+\mathcal{O}\left(r^{-1}\right)\right) .
$$

If $\cos k \Theta_{j} \neq 0$ (equivalently, if $\Theta_{j}$ does not coincide with any angle $\theta_{l}, l=$ $1, \ldots, 2 k)$, then $x_{3}$ diverges monotonically to infinity as $r \rightarrow \infty$. Such is the case, for instance, when $n=k$ and $a_{k} \in i \mathbb{R}$. In the case $\cos k \Theta_{j}=0$, L'Hôpital rule implies $\frac{\cos \left(n \theta_{j}^{A}(r)+\alpha_{n}\right)}{\cos k \theta_{j}^{A}(r)} \rightarrow \frac{n}{k}$ when $r \rightarrow \infty$, hence $\cos k \theta_{j}^{A}(r)$ has the same behavior as $\cos \left(n \theta_{j}^{A}(r)+\alpha_{n}\right)$, and using (12) we obtain

$$
\begin{aligned}
x_{3}\left(\Gamma_{j}^{A}(r)\right) & \sim \frac{1}{n} r^{k} \cos \left(n \theta_{j}^{A}(r)+\alpha_{n}\right) \\
& =\frac{A}{n\left|a_{n}\right|} r^{k-n}-\frac{m}{n\left|a_{n}\right|} r^{k-n} \log r-\sum_{\lambda=-\infty}^{n-1} \frac{\left|a_{\lambda}\right|}{n\left|a_{n}\right|} \cos \left(\lambda \theta_{j}^{A}(r)+\alpha_{\lambda}\right) r^{k-n+\lambda} .
\end{aligned}
$$

Recall that the intersection of $X(D(\infty, R))$ with the upper halfspace $\left\{x_{3}>0\right\}$ is the union of the images through the immersion $X$ of the sectors $S_{1}, S_{3}, \ldots, S_{2 k-1}$. Each one of these images will be called an upper leaf of the end. Similarly, lower leaves are defined as the images by $X$ of the even sectors. Given an upper leaf $X\left(S_{l}\right)$ (l odd), we define a quasivertical axis of $X\left(S_{l}\right)$ as a proper arc $\zeta \subset X\left(S_{l}\right)$ that verifies the following condition: there exists a vertical halfline $t \subset\left\{x_{3} \geq 0\right\}$ with boundary at height zero such that for any tubular neighborhood $T$ of $t$, there exists a height $h>0$ such that $\zeta \cap\left\{x_{3}>h\right\}$ is contained in $T$ and $\zeta \cap\left\{x_{3} \leq h\right\}$ is compact. A quasivertical axis of a lower leaf $X\left(S_{l}\right)$ (with $l$ even) can be defined as well, exchanging the upper halfspace by the lower one.

We now state the relationship between preimages by the Gauss map of horizontal parallels of the sphere and these quasivertical axes.

Proposition 3. Let $X: D(\infty, R) \longrightarrow \mathbb{R}^{3}$ be a minimal immersion with the normalization in Section 2 Suppose that $n=p k$, with $p \geq 1$ integer.

i) If $p \geq 2$, then there exists an integer $\mu$ with $0 \leq \mu \leq 2 k$, such that for each $A \in \mathbb{R}$, the set $X\left(|g|^{-1}\left(e^{A}\right)\right)$ consists of $2 n-\mu$ quasivertical axes and $\mu$ nonproper curves. Moreover, each leaf contains at least $p-1$ of these quasivertical axes, and if $\mu<2 k$, then there exists a leaf $X\left(S_{l}\right)$ such that $X\left(|g|^{-1}\left(e^{A}\right) \cap S_{l}\right)$ contains at least $p$ quasivertical axes. 
ii) If $n=k, a_{k} \in i \mathbb{R}$ and either $n \geq 2$ or $(n, m)=(1,0)$, then the image by $X$ of $|g|^{-1}(1)$ consists of $2 k$ quasivertical axes, one per leaf.

Proof. Fix $A \in \mathbb{R}$ and consider a curve $\Gamma_{j}^{A} \subset D(\infty, R)$ in $|g|^{-1}\left(e^{A}\right), j=1, \ldots, 2 n$.

Suppose first that $p \geq 2$. From (13) and Lemma 1 (use $(\delta, a, b)=(0,0, n / k)$ if $\sinh \left(A+i k \Theta_{j}\right) \neq 0$; if on the contrary $\sinh \left(A+i k \Theta_{j}\right)=0$, then we have $(\delta, b)=(0, n / k)$ while $a \leq-1 / k$, so $a+1<b$ always holds), we conclude that $\left(x_{1}+i x_{2}\right)\left(\Gamma_{j}^{A}\right)$ is a spiral that converges to a point $q \in \mathbb{C}$. As the $2 n$ curves $\Gamma_{j}^{A}$ are asymptotically rays forming an equiangular system at infinity of angle $\pi / n$ and the boundaries of the sectors $S_{l}(l=1, \ldots, 2 k)$ have an analogous distribution with angle $\pi / k$, it follows that the number of angles $\Theta_{j}$ such that $\theta_{l-1}<\Theta_{j} \leq \theta_{l}$ for a given $l$ is exactly $p$. Consider one of the curves $X\left(\Gamma_{j}^{A}\right)$ with $\theta_{l-1}<\Theta_{j}<\theta_{l}$. As $\cos k \Theta_{j} \neq 0$, it follows from the argument just before this proposition that $x_{3}\left(\Gamma_{j}^{A}(r)\right)$ diverges monotonically to infinity as $r \rightarrow \infty$. Thus $X\left(\Gamma_{j}^{A}\right)$ is eventually contained in any tubular neighborhood of the halfline above (or below) $q$, that is, $X\left(\Gamma_{j}^{A}\right)$ is a quasivertical axis. As we have at least $p-1$ angles $\Theta_{j}$ with $\theta_{l-1}<\Theta_{j}<$ $\theta_{l}$, it follows that each leaf contains at least $p-1$ quasivertical axes. We now have two possibilities: first, suppose that the angles $\theta_{l}$ never coincide with the angles $\Theta_{j}$. In this setting, the $2 n$ curves $X\left(\Gamma_{j}^{A}\right)$ are quasivertical axes and $i$ ) holds with $\mu=0$. The second possibility is that each angle $\theta_{l}$ coincides with one of the angles $\Theta_{j}$. In this case, the number of angles $\Theta_{j}$ with $\theta_{l-1}<\Theta_{j}<\theta_{l}$ is $p-1$, and each corresponding $X\left(\Gamma_{j}^{A}\right)$ is a quasivertical axis. It remains to analyze the asymptotic behavior of the $2 k$ curves $X\left(\Gamma_{j}^{A}\right)$ where $\cos k \Theta_{j}=0$. We call $\mu$ to the number of such curves that have $\left.x_{3}\right|_{\Gamma_{j}^{A}}$ bounded (thus $0 \leq \mu \leq 2 k$ ). Equation (14) insures that whenever $\left.x_{3}\right|_{\Gamma_{j}^{A}}$ is bounded, it must converge monotonically (after possibly discarding a compact subarc of $\left.\Gamma_{j}^{A}\right)$ to a certain height, and the curve $X\left(\Gamma_{j}^{A}\right)$ is not proper. We now show that if $\left.x_{3}\right|_{\Gamma_{j}^{A}}$ is unbounded, then it eventually diverges monotonically to $\pm \infty$ (thus $X\left(\Gamma_{j}^{A}\right)$ is again a quasivertical axis). As $p \geq 2$, the two first terms in the right-hand side of (14) decay to zero as $r \rightarrow \infty$. As $\left.x_{3}\right|_{\Gamma_{j}^{A}}$ is not bounded, it follows also from (14) that there exists a largest integer $\lambda$ with $n-k<\lambda \leq n-1$ such that $\left|a_{\lambda}\right| \cos \left(\lambda \Theta_{j}+\alpha_{\lambda}\right) \neq 0$ and therefore, $x_{3}\left(\Gamma_{j_{0}}^{A}(r)\right)$ diverges monotonically to $\pm \infty$.

We now prove that there exists a leaf which contains at least $p$ of these quasivertical axes, provided that $\mu<2 k$. First, note that this strict inequality insures that there exist $j \in\{1, \ldots, 2 n\}$ and $l \in\{1, \ldots, 2 k\}$ with $\Theta_{j}=\theta_{l}$ such that $X\left(\Gamma_{j}^{A}\right)$ is a quasivertical axis. As the boundary of each leaf lies at height zero, it follows that $X\left(\Gamma_{j}^{A}\right)$ is eventually disjoint of the common boundary of the leaves $X\left(S_{l}\right), X\left(S_{l-1}\right)$, so, in particular, $X\left(\Gamma_{j}^{A}\right)$ is eventually contained in one of these two leaves, say $X\left(S_{l}\right)$. As this last leaf has $p-1$ quasivertical axes $X\left(\Gamma_{h}^{A}\right)$ with $\theta_{l-1}<\Theta_{h}<\theta_{l}$, we deduce that $X\left(S_{l}\right)$ contains, at least, $p$ quasivertical axes. Thus $\left.i\right)$ is proved.

Finally, assume that $n=k, a_{k} \in i \mathbb{R}$ and either $n \geq 2$ or $(n, m)=(1,0)$. The condition on $a_{k}$ guarantees that $\sinh \left(A+i k \Theta_{j}\right)=0$ if we take $A=0$; thus, we can use Lemma 1 on $\Gamma_{j}^{0}$ with $(\delta, b)=(0,1), a \leq-1 / k$, concluding again that the horizontal projection of $X\left(\Gamma_{j}^{0}\right)$ is a spiral that converges to a point. $x_{3}$ diverges monotonically to $\pm \infty$ because $\cos k \Theta_{j} \neq 0$ when $n=k$ and $a_{k} \in i \mathbb{R}$, as we remarked before this proposition. Note that the condition $\cos k \Theta_{j} \neq 0$ also implies that the angles $\Theta_{j}$ do not coincide with the angles $\theta_{l}$, hence there exists exactly one quasivertical axis in each leaf. 
We now study the preimages by the Gauss map of vertical meridians of the sphere. As this study shares some aspects with the preceding one of horizontal spherical parallels, we will only develop the new properties in detail.

The set $\{\operatorname{Imag}(H(z))=0\} \subset D(\infty, R)$ consists of $2 n$ divergent curves asymptotic to the rays of argument $\Theta_{j}+\frac{\pi}{2 n}, j=1, \ldots, 2 n$, which form an equiangular system centered at $\infty$ that bisects the one that appeared with the horizontal parallels. Similarly, the set $\{\arg (g)=B\} \subset D(\infty, R)$ for any $B \in \mathbb{R}$ consists of $2 n$ divergent curves $\widehat{\Gamma}_{1}^{B}, \ldots, \widehat{\Gamma}_{2 n}^{B}$ asymptotic to the rays of angle $\Theta_{j}+\frac{\pi}{2 n}, j=1, \ldots, 2 n$. In order to describe the geometry of $X\left(\widehat{\Gamma}_{j}^{B}\right)$, first we parametrize the curve in the $z$-plane as $\widehat{\Gamma}_{j}^{B}(r)=r e^{i \widehat{\theta}_{j}^{B}(r)}$. Substituting the expansion of $H$ into the condition $\left.\arg (g)\right|_{\widehat{\Gamma}_{j}^{B}}=B$, we obtain $\sin \left(n \widehat{\theta}_{j}^{B}(r)+\alpha_{n}\right)=-\frac{m \widehat{\theta}_{j}^{B}(r)}{\left|a_{n}\right| r^{n}}+\mathcal{O}\left(r^{-1}\right)$, hence by derivation $\left(\widehat{\theta}_{j}^{B}\right)^{\prime}(r)=\mathcal{O}\left(r^{-2}\right)$, which implies that $\widehat{\theta}_{j}^{B}(r)=\Theta_{j}+\frac{\pi}{2 n}+\mathcal{O}\left(r^{-1}\right)$. In terms of the parameter $s=r^{k}$, our curve is $\widehat{\Gamma}_{j}^{B}(s)=s^{1 / k} e^{i\left[\Theta_{j}+\frac{\pi}{2 n}+\mathcal{O}\left(s^{-1 / k}\right)\right]}$, and using (4) we obtain

$$
\begin{aligned}
& \frac{d}{d s}\left(\left(x_{1}+i x_{2}\right)\left(\widehat{\Gamma}_{j}^{B}\right)\right) \\
& \quad=\frac{1}{2 k}\left[e^{-A(s)-i k\left(\Theta_{j}+\frac{\pi}{2 n}\right)}-e^{A(s)+i k\left(\Theta_{j}+\frac{\pi}{2 n}\right)}\right]\left[1+\mathcal{O}\left(s^{-1 / k}\right)\right] e^{i B},
\end{aligned}
$$

where $A(s)=A\left(\widehat{\Gamma}_{j}^{B}(s)\right)$. But

$$
\begin{aligned}
A\left(\widehat{\Gamma}_{j}^{B}(r)\right) & =m \log r+\operatorname{Real}(H) \\
& =r^{n}\left[\left|a_{n}\right| \cos \left(n \Theta_{j}+\frac{\pi}{2}+\alpha_{n}\right)+m \frac{\log r}{r^{n}}+\mathcal{O}\left(r^{-1}\right)\right]
\end{aligned}
$$

which can be written as $r^{n}\left[\left|a_{n}\right| \cos \left(n \Theta_{j}+\frac{\pi}{2}+\alpha_{n}\right)+\mathcal{O}\left(r^{-1}\right)\right]$ if we assume $n \geq 2$ (note that $\cos \left(n \Theta_{j}+\frac{\pi}{2}+\alpha_{n}\right)= \pm 1$ ), and thus $A\left(\widehat{\Gamma}_{j}^{B}(s)\right)=s^{n / k}\left[C+\mathcal{O}\left(s^{-1 / k}\right)\right]$ with $C \in \mathbb{R}-\{0\}$. Now a straightforward computation gives $\frac{d}{d s}\left(\left(x_{1}+i x_{2}\right)\left(\widehat{\Gamma}_{j}^{B}\right)\right)=$ $r(s) e^{i \varphi(s)}$ in polar coordinates, with

$$
r(s)=\left[\frac{1}{2 k}+\mathcal{O}\left(s^{-1 / k}\right)\right] e^{s^{n / k}\left[C_{2}+\mathcal{O}\left(s^{-1 / k}\right)\right],} \quad \varphi(s)=\beta+\mathcal{O}\left(s^{-1 / k}\right),
$$

for certain $\beta, C_{2} \in \mathbb{R}, C_{2}>0$-although we will not use them, the expressions for these constants are $C_{2}=\left|a_{n}\right|$ and $\beta=k\left(\Theta_{j}+\frac{\pi}{2 n}\right)+B+\pi$ (resp. $\beta=$ $-k\left(\Theta_{j}+\frac{\pi}{2 n}\right)+B$ ) if $\cos \left(n \Theta_{j}+\frac{\pi}{2}+\alpha_{n}\right)=1$ (resp. if $\cos \left(n \Theta_{j}+\frac{\pi}{2}+\alpha_{n}\right)=-1$ ). Using Lemma 2, the horizontal projection of $X\left(\widehat{\Gamma}_{j}^{B}\right)$ is a divergent curve whose argument converges to $\beta$. As in the study of the quasivertical axes, the height of $X\left(\widehat{\Gamma}_{j}^{B}\right)$ is given by $x_{3}\left(\widehat{\Gamma}_{j}^{B}(r)\right)=\frac{1}{k} r^{k} \cos k \widehat{\theta}_{j}^{B}(r)$, which diverges monotonically provided that $\cos k\left(\Theta_{j}+\frac{\pi}{2 n}\right) \neq 0$.

Given an upper or lower leaf $X\left(S_{l}\right)$, we define an oblique ray of $X\left(S_{l}\right)$ as an arc $\zeta=\left(\zeta_{1}, \zeta_{2}, \zeta_{3}\right) \subset X\left(S_{l}\right)$ such that $\zeta_{1}^{2}+\zeta_{2}^{2}$ is proper and $\arg \left(\zeta_{1}+i \zeta_{2}\right)$ converges to a fixed angle as $\zeta$ diverges. With this definition we can deduce the following statement from the previous arguments and the ideas in the proof of Proposition 3

Proposition 4. Let $X: D(\infty, R) \longrightarrow \mathbb{R}^{3}$ a minimal immersion with the normalization in Section 2 Suppose that $n \geq 2$ and $n=p k$ with $p \in \mathbb{N}$. Then, for each 
$B \in \mathbb{R}$ the image by $X$ of the set $\arg (g)^{-1}(B)$ consists of $2 n$ oblique rays, and each leaf contains at least $p-1$ of them.

We finish this section with another criterion of self-intersection which applies in a particular nonproper setting.

Proposition 5. Let $X: D(\infty, R) \longrightarrow \mathbb{R}^{3}$ be a minimal immersion with the normalization in Section 圆. Suppose that $n=2 k$ and that the number of nonproper curves in $X\left(|g|^{-1}\left(e^{A}\right)\right)$ is $\mu=2 k$, where $A \in \mathbb{R}$ (see Proposition [3). Then, $X$ is not an embedding.

Proof. Applying the arguments in the proof of Proposition 3 we conclude that given $A \in \mathbb{R}$, the set $X\left(|g|^{-1}\left(e^{A}\right)\right)$ consists of $2 k$ quasivertical axes, one in each leaf, and all the angles $\theta_{l}$ coincide with the angles $\Theta_{j}$ of a given parity (say odd), $\theta_{l}=\Theta_{2 l+1}$ for each $l$. We now show how our hypotheses constrain the Gauss map $g(z)=z^{m} e^{H(z)}$ to an explicit form.

By definition of $\Theta_{j}$ and $\theta_{l}$, we have $0=\cos \left(2 k \Theta_{2 l+1}+\alpha_{2 k}\right)=\cos \left(2 k \theta_{l}+\alpha_{2 k}\right)=$ $-\cos \alpha_{2 k}$, thus $a_{2 k} \in i \mathbb{R}$. On the other hand, the boundness of $\left.x_{3}\right|_{\Gamma_{j}^{A}}$ for all $j$ odd together with equation (14) give that $\left|a_{\lambda}\right| \cos \left(\lambda \theta_{l}+\alpha_{\lambda}\right)=0$ for all $\lambda=$ $k+1, \ldots, 2 k-1$ and all $l=1, \ldots, 2 k$. As none of such $\lambda$ is a multiple of $k$, a similar argument to the one in Lemma $4 i$ ) but exchanging sine for cosine shows that it must hold $a_{k+1}=\ldots=a_{2 k-1}=0$.

Fix $l \in\{1, \ldots, 2 k\}$ odd (i.e. $X\left(S_{l}\right)$ is an upper leaf) and consider the nonproper curve $X\left(\Gamma_{2 l+1}^{A}\right)$. We showed in the proof of Proposition 3 that $\left(x_{1}+i x_{2}\right)\left(\Gamma_{2 l+1}^{A}\right)$ is a spiral that converges to a point $q \in \mathbb{C}$, while its third coordinate converges monotonically (after possibly removing a compact subarc) to a finite height $c_{0} \geq$ 0 , depending on $l$. Moreover, the monotonocity of $\left.x_{3}\right|_{\Gamma_{2 l+1}^{A}}$ allows us to assume without loss of generality that $\Gamma_{2 l+1}^{A} \subset S_{l}$ after possibly discarding a compact subarc (otherwise, $c_{0}=0$ and we exchange $S_{l}$ by an adjacent lower leaf $S_{l+1}$ ). We now study the geometry of the horizontal level curves $X\left(\gamma_{c, l}\right)$ as $\arg \left(\gamma_{c, l}\right)$ tends to $\theta_{l}$, for all $c>0$ with $\left|c-c_{0}\right|$ small enough and $c \neq c_{0}$. As $c \neq 0$, we can use the parametrization $\gamma_{c, l}(s)=(k c s)^{1 / k} e^{i \theta(s)}$ with $s=\frac{1}{\cos k \theta(s)}$, hence (8) becomes

$$
\begin{aligned}
A\left(\gamma_{c, l}(s)\right)= & \frac{m}{k} \log (k c s)+\mathcal{O}\left(s^{1-\frac{1}{k}}\right)+\left|a_{k}\right| k c s \cos \left(k \theta(s)+\alpha_{k}\right) \\
& +\left|a_{2 k}\right|(k c s)^{2} \cos \left(2 k \theta(s)+\alpha_{2 k}\right) \\
= & \frac{m}{k} \log (k c s)+\mathcal{O}\left(s^{1-\frac{1}{k}}\right) \\
& +c s\left[\left|a_{k}\right| k \cos \left(k \theta_{l}+\alpha_{k}\right)-2\left|a_{2 k}\right| k^{2} c \frac{\sin \left(2 k \theta_{l}+\alpha_{2 k}\right)}{\sin k \theta_{l}}\right] \\
= & \frac{m}{k} \log (k c s)+\mathcal{O}\left(s^{1-\frac{1}{k}}\right)-k c s \sin k \theta_{l}\left[\left|a_{k}\right| \sin \alpha_{k}-2 k c\left|a_{2 k}\right| \sin \alpha_{2 k}\right] .
\end{aligned}
$$

Note that the expression enclosed in brackets in the last equation vanishes, at most, for one nonzero value of $c$ (that could be $c_{0}$ ), hence there exists $\varepsilon>0$ such that the growth of $A\left(\gamma_{c, l}(s)\right)$ for all $c>0$ with $\left|c-c_{0}\right| \leq \varepsilon$ and $c \neq c_{0}$ is as $s$ times a nonzero constant (and this argument works for all $l=1, \ldots, 2 k$, so we can choose 
such $\varepsilon>0$ independent of $l$ ). Analogously, equation (9) says

$$
\begin{aligned}
B\left(\gamma_{c, l}(s)\right) & =m \theta(s)+\mathcal{O}(s)+\left|a_{2 k}\right|(k c s)^{2} \sin \left(2 k \theta(s)+\alpha_{2 k}\right) \\
& =m \theta_{l}+\mathcal{O}(s)-\left|a_{2 k}\right|(k c s)^{2} \sin \alpha_{2 k} .
\end{aligned}
$$

These properties together with equation (7) insure that for all $c>0$ with $\left|c-c_{0}\right| \leq \varepsilon$ and $c \neq c_{0}$, the curve $X\left(\gamma_{c, l}\right)$ satisfies the requirements of Lemma1 1 i) when $\theta \rightarrow \theta_{l}$ and therefore, it must be a proper spiral (note that the last equation implies that we lie in case $i$ ) of Claim 2 with exponent $b\left(\theta_{l}\right)=2$ for each such $c$ ), and this holds for all $l$. If we focus on the level curve at height zero (as we are working with values of $c$ close enough to $c_{0}$, the height zero should be taken into account only when $\left.c_{0}=0\right)$, our hypotheses $a_{k+1}=\ldots=a_{2 k-1}=\cos \left(2 k \theta_{l}+\alpha_{2 k}\right)=0$ constrain (3i) to the simpler form

$$
\begin{gathered}
A\left(\gamma_{0, l}(s)\right)=\frac{m}{k} \log s+\mathcal{O}\left(s^{1-\frac{1}{k}}\right)-\left|a_{k}\right| s \sin k \theta_{l} \sin \alpha_{k}, \\
B\left(\gamma_{0, l}(s)\right)=m \theta_{l}+\mathcal{O}(s)-\left|a_{2 k}\right| s^{2} \sin \alpha_{2 k} .
\end{gathered}
$$

Our next goal consists of proving that if $X$ is an embedding, then $X\left(\gamma_{c_{0}, l}\right)$ is a nonproper spiral when $\theta \rightarrow \theta_{l}$, for all $l=1, \ldots, 2 k$ (as $\gamma_{0, l}$ cannot be parametrized by the argument $\theta$, when $c_{0}=0$ the notation $\theta \rightarrow \theta_{l}$ has the obvious meaning $\left.\left|\gamma_{c_{0}, l}\right| \rightarrow+\infty\right)$. Given $l$ fixed, as the argument of $\frac{d}{d s}\left(x_{1}+i x_{2}\right)\left(\gamma_{c_{0}, l}\right)$ grows as $s^{2}$ times a nonzero constant (even in the case $c_{0}=0$ ), Lemma 1 insures that $X\left(\gamma_{c_{0}, l}\right)$ is a spiral at height $c_{0}$ when $\theta \rightarrow \theta_{l}$, which may be divergent, asymptotic to a circle or to a point depending on the values of $\delta$ and $a$ in the expansion of the modulus of its velocity vector. Recall that we have proved that $X\left(\Gamma_{2 l+1}^{A}(r)\right)$ converges to $\left(q, c_{0}\right) \in \mathbb{C} \times \mathbb{R}$ as $r \rightarrow+\infty$, and there exists $\varepsilon>0$ such that $X\left(\gamma_{c, l}\right)$ is a proper spiral when $\theta \rightarrow \theta_{l}$ for all $c \neq c_{0}$ with $\left|c-c_{0}\right| \leq \varepsilon$. Let $C_{1}$ be a vertical cylinder with axis $q+\mathbb{R} e_{3}$ (here $\left.e_{3}=(0,0,1)\right)$ and radius $R_{1}>0$ large. After discarding a compact subarc, we can suppose that $X\left(\Gamma_{2 l+1}^{A}\right)$ is completely contained in the compact solid cylinder $U$ enclosed by $C_{1} \cup\left\{\left|x_{3}-c_{0}\right|=\varepsilon\right\}$. Reasoning by contradiction, assume that $X$ is an embedding and $X\left(\gamma_{c_{0}, l}\right)$ is a proper spiral when $\theta \rightarrow \theta_{l}$. We now divide the argument in two cases: first assume that $c_{0}>0$. As $X\left(\gamma_{c_{0}, l}\right)$ is proper, it must intersect $C_{1}$ in at least one point $P_{c_{0}}$. We can also suppose without loss of generality that $C_{1}$ cuts to $X(D(\infty, R))$ transversally at $P_{c_{0}}$. Let us call $\beta$ the connected component of $X(D(\infty, R)) \cap C_{1}$ containing to $P_{c_{0}}$. This curve $\beta$ is not horizontal al $P$ (otherwise, the tangent plane of the surface at $P_{c_{0}}$ would be horizontal, a contradiction), thus by shrinking $\varepsilon$ we can assume that $\beta$ cuts any horizontal plane at height $c \in\left[c_{0}-\varepsilon, c_{0}+\varepsilon\right]$ at a point $P_{c}$. On the other hand, as the branch of $X\left(\gamma_{c_{0}, l}\right)$ as $\theta \rightarrow \theta_{l}$ is a proper spiral, we can choose $R_{1}$ large enough so that there exists a compact subarc $\varrho_{c_{0}}$ of such a branch starting at $P_{c_{0}}$ with the following two properties:

- $\varrho_{c_{0}}-\left\{P_{c_{0}}\right\}$ is contained in the exterior of the disk enclosed by $C_{1} \cap\left\{x_{3}=c_{0}\right\}$,

- $\varrho_{c_{0}}$ winds three complete turns around $C_{1} \cap\left\{x_{3}=c_{0}\right\}$ (i.e. the argument of its velocity vector covers thrice the unit circle).

By continuity, $\varepsilon$ can be taken small enough such that forall $c>0$ with $\left|c-c_{0}\right| \leq \varepsilon$, there exists a compact subarc $\varrho_{c}$ of the branch of $X\left(\gamma_{c, l}\right)$ as $\theta \rightarrow \theta_{l}$ such that $\varrho_{c}$ starts at $P_{c}$, it is completely contained in the exterior of the disk enclosed by $C_{1} \cap\left\{x_{3}=c\right\}$ and $\varrho_{c}$ winds two complete turns around $C_{1} \cap\left\{x_{3}=c_{0}\right\}$. Choose $R_{2}>$ $R_{1}$ big enough so that the vertical cylinder with axis $q+\mathbb{R} e_{3}$ and radius $R_{2}$ encloses the (compact) union $\Omega$ of all these curves $\varrho_{c}$ with $\left|c-c_{0}\right| \leq \varepsilon$ (we can think about 
$\Omega$ as a 'compact spiraling cylinder' inside the surface, which necessarily intersects any curve trapped between heights $c_{0}-\varepsilon$ and $c_{0}+\varepsilon$ having one of its extrema inside $C_{1}$ and the other one outside $C_{2}$, whose tangent vector has a horizontal projection not spanning an angle bigger than $\pi$ ). On the other hand, note that both $\Gamma_{2 l+1}^{A}, \gamma_{c_{0}+\varepsilon, l} \subset S_{l}$ are proper arcs whose arguments tend to $\theta_{l}$, and they are eventually disjoint (the $x_{3}$-function goes to $c_{0}$ on $\Gamma_{2 l+1}^{A}$ and is constantly $c_{0}+\varepsilon$ on $\left.\gamma_{c_{0}+\varepsilon, l}\right)$. For large values of $B \in \mathbb{R}$ there exists a compact $\operatorname{arc} \widehat{\Gamma}^{B} \subset S_{l}$ starting at a point of $\Gamma_{2 l+1}^{A}$ and ending at a point of $\gamma_{c_{0}+\varepsilon, l}$, which applies by $X$ on a piece of an oblique ray $\zeta$ with $\arg (g) \equiv B$ whose third component $\zeta_{3}$ increases monotonically from $x_{3}\left(\widehat{\Gamma}^{B} \cap \Gamma_{2 l+1}^{A}\right)$ (which tends to $c_{0}$ as $\left.B \rightarrow+\infty\right)$ to $x_{3}\left(\widehat{\Gamma}^{B} \cap \gamma_{c_{0}+\varepsilon, l}\right)=c_{0}+\varepsilon$. In particular, $\zeta$ is trapped between heights $c_{0}-\varepsilon$ and $c_{0}+\varepsilon$. By taking $B$ large enough, the extrema of $\zeta$ are one inside the small cylinder $C_{1}$ and the other one outside the bigger one $C_{2}$, and the argument of the horizontal projection of the tangent vector to $\zeta$ does not span more than $\pi$ (the horizontal slab where $\zeta$ is trapped can be chosen of arbitrarily small height, so $\zeta$ is extremely close to a horizontal curve and its tangent vector horizontally projected spans an angle as close as we want to the angle spanned by the horizontal projection of the Gauss map of the surface along this curve, which is zero). Thus $\zeta$ must intersect $\Omega$. But this contradicts the embeddedness of $X$, as $\Omega$ is a fixed compact subset on the surface and $B$ can be chosen arbitrarily large.

In the case $c_{0}=0$, the same argument holds with minor modifications. In summary, $X\left(\gamma_{c_{0}, l}\right)$ is a nonproper spiral when $\theta \rightarrow \theta_{l}$, for all $l=1, \ldots, 2 k$.

We now show that $a_{1}=\ldots=a_{k-1}=0$. Reasoning by contradiction, otherwise we can choose the maximum $\lambda \in\{1, \ldots, k-1\}$ such that $a_{\lambda} \neq 0$. Using again Lemma 4 i) with $k, \lambda$ and $\alpha=\alpha_{\lambda}$ but exchanging sine for cosine, one can find $l \in\{1, \ldots, 2 k\}$ such that $\cos \left(\lambda \theta_{l}+\alpha_{\lambda}\right) \neq 0$. But the term $\mathcal{O}\left(s^{1-\frac{1}{k}}\right)$ in equation (15) for $c=c_{0} \neq 0$ (resp. in equation (17) for $c=c_{0}=0$ ) contains the term $\left|a_{\lambda}\right|(k c s)^{\lambda / k} \cos \left(\lambda \theta(s)+\alpha_{\lambda}\right)$ (resp. $\left.\left|a_{\lambda}\right| s^{\lambda / k} \cos \left(\lambda \theta_{l}+\alpha_{\lambda}\right)\right)$, thus $A\left(\gamma_{c_{0}, l}(s)\right)$ grows at least as $s^{\lambda / k}$. This fact contradicts that $X\left(\gamma_{c_{0}, l}\right)$ is a nonproper spiral when $\theta \rightarrow \theta_{l}$ (in the notation of Lemma 1 the modulus $r(s)$ of the velocity vector of $X\left(\gamma_{c_{0}, l}\right)$ would have $\left.\delta \geq \frac{\lambda}{k}>0\right)$.

As $X\left(\gamma_{c_{0}, l}\right)$ is a nonproper spiral, it must be asymptotic to a circle or to a point (cases ii) and iii) of Lemma 11). Next we prove that if $X\left(\gamma_{c_{0}, l}\right)$ converges to a point, then $X\left(\gamma_{c, l}\right)$ cannot be embedded for $c \neq c_{0}$ close enough to $c_{0}$. Whenever $\left|c-c_{0}\right| \leq \varepsilon$, the spiral $X\left(\gamma_{c, l}\right)$ rotates as $\theta \rightarrow \theta_{l}$ (again with the previous abuse of notation if $\left.c_{0}=0\right)$ in a fixed direction that depends on whether $B(c, s)=B\left(\gamma_{c, l}(s)\right)$ diverges to $+\infty$ or to $-\infty$ (see equation (7) for the case $c \neq 0$ and equation (5) for the case $c=c_{0}=0$ ). As even at height $c_{0}$ we have a well-defined winding direction and $\frac{\partial B}{\partial s}$ is continuous, we can assume after possibly shrinking $\varepsilon>0$ that there exists $R_{1}>0$ such that none of the branches as $\theta \rightarrow \theta_{l}$ of the curves $X\left(\gamma_{c, l}\right)$, $\left|c-c_{0}\right| \leq \varepsilon$, turns its winding direction in the proper subarc $\left|\gamma_{c, l}\right| \geq R_{1}$. Denote by $\theta \in[\nu, \rho] \mapsto X\left(\gamma_{c_{0}, l}\right)(\theta)$ a compact subarc of the branch of $X\left(\gamma_{c_{0}, l}\right)$ as $\theta \rightarrow \theta_{l}$, suitable chosen so that

- $\left|\gamma_{c_{0}, l}(\theta)\right|>R_{1}$ for all $\theta \in[\nu, \rho]$,

- $X\left(\gamma_{c_{0}, l}([\nu, \rho])\right)$ winds three complete turns around its limit point (here $\theta_{l}-\sigma<$ $\nu<\rho<\theta_{l}$ for a small $\sigma>0$ and we maintain the abuse of notation that exchanges angle by modulus in the case $c_{0}=0$ ). 
By continuity, we can increase the height to $c=c_{0}+\varepsilon_{1}$ with $\varepsilon_{1} \in(0, \varepsilon)$, so that we find a compact subarc $\theta \in[\nu, \rho] \mapsto X\left(\gamma_{c, l}(\theta)\right)$ of the branch of $X\left(\gamma_{c, l}\right)$ as $\theta \rightarrow \theta_{l}$ with the properties $\left|\gamma_{c, l}(\theta)\right|>R_{1}$ for all $\theta \in[\nu, \rho]$ and $X\left(\gamma_{c, l}([\nu, \rho])\right)$ winds two complete turns (i.e. the argument of its velocity vector covers twice the unit circle). As $X\left(\gamma_{c_{0}, l}\right)$ converges to a point as $\theta \rightarrow \theta_{l}$, it 'winds towards inside' (i.e. the distance of $X\left(\gamma_{c_{0}, l}(\theta)\right)$ to the limit point eventually decreases monotonically), hence the same holds with the compact subarc $\theta \in[\nu, \rho] \mapsto X\left(\gamma_{c_{0}, l}(\theta)\right)$. By taking $\varepsilon_{1}$ small enough, we can assume without loss of generality that the compact subarc $\theta \in[\nu, \rho] \mapsto X\left(\gamma_{c, l}(\theta)\right)$ also 'winds towards inside'. This property, together with the facts that $X\left(\gamma_{c, l}\right)$ is a proper spiral and it cannot turn its winding direction in $\left[\rho, \theta_{l}\right)$ imply that $\left.X\left(\gamma_{c, l}\right)\right|_{[\nu, \rho]}$ must intersect to $\left.X\left(\gamma_{c, l}\right)\right|_{\left[\rho, \theta_{l}\right)}$, and we have the desired selfintersection (note that this argument also works if the branch of $X\left(\gamma_{c_{0}, l}\right)$ as $\theta \rightarrow \theta_{l}$ lies in case ii) of Lemma 1 and the distance of $X\left(\gamma_{c_{0}, l}\right)$ to the center of its limit circle still decreases monotonically).

Finally, we analyze the case in which $X\left(\gamma_{c_{0}, l}\right)$ is a spiral converging to a nondegenerate circle. Roughly speaking, we consider the level curves in the same sector close to (but different from) the nonproper height $c_{0}$ : they consist asymptotically of two divergent spirals (possibly inside a connected level curve), both turning the same way (otherwise, the desired self-intersection would be obvious). However, they do not grow at the same speed. We will prove that one of the spirals grows sufficiently faster than the other one, so both cannot be imbricated. First, as $a_{1}=\ldots=a_{k-1}=0$, equation (15) becomes

$$
A\left(\gamma_{c, l}(s)\right)=\frac{m}{k} \log (k c s)+\mathcal{O}(1)-k c s \sin k \theta_{l}\left[\left|a_{k}\right| \sin \alpha_{k}-2 k c\left|a_{2 k}\right| \sin \alpha_{2 k}\right],
$$

for the branch of the level curve at height $c \neq 0, c_{0}$ as $\theta \rightarrow \theta_{l}$, while equation (16) remains unchanged. For the other branch $\left(\theta \rightarrow \theta_{l-1}\right)$ at the same height coming from the same sector $S_{l}$, we need only to change the limit angular value $\theta_{l}$ by $\theta_{l-1}$, thus the sign of the coefficient in the $s^{2}$-term of $B$ remains (both spirals turn the same way) and the same holds with the one in the logarithmic term of $A$, but the main $s$-term of $A$ changes sign. In terms of the notations in Lemma 1 this means that we have $\delta=1, b=2$ at both branches but $a$ switches sign. More precisely, the modulus of the velocity of these two curves are described by the model $r(s)=s^{a}\left[C_{1}+\mathcal{O}\left(s^{-1 / k}\right)\right] e^{s\left[C_{2}+\mathcal{O}\left(s^{-1 / k}\right)\right]}$ with the same constant $C_{2}$ and opposite values of $a=a(c)$ (namely $m / k$ and $-m / k$ ).

On the other hand, as $X\left(\gamma_{c_{0}, l}\right)$ lies in case ii) of Lemma11 and equation (17) says that $b\left(c_{0}\right)$ also equals 2 , it follows that $|m|=k$, so $a\left(c_{0}\right)= \pm 1$.

We now study the 'diameter' of the spiral $X\left(\gamma_{c, l}\right)$ as $\theta \rightarrow \theta_{l}$, for $c \neq 0, c_{0}$. The arguments that follow are based on (and use the notations in) the proof of Lemma 1 see Appendix. Given $\alpha \in[0,2 \pi)$, the function $x_{\alpha}(s)=\left\langle X\left(\gamma_{c, l}\right)(s), e^{i(\alpha+\pi / 2)}\right\rangle(s>0$ large), has an infinite countable number of critical points passing consecutively from local maxima (denoted by $s_{2 n}$ ) to local minima $\left(s_{2 n-1}\right)$. By the diameter of the spiral $X\left(\gamma_{c, l}\right)$, we mean the absolute difference between consecutive critical points of $x_{\alpha}$, which can be measured by the integral

$$
\left|\Delta_{n}\right|=\left|x_{\alpha}\left(s_{n+1}\right)-x_{\alpha}\left(s_{n}\right)\right|=\int_{n \pi}^{(n+1) \pi} h(u+\alpha)|\sin u| d u,
$$

where $h(u)=r(\psi(u)) \psi^{\prime}(u), r=r(s)$ is the modulus of the velocity vector of $X\left(\gamma_{c, l}\right)$ and $\psi(u)=s$ is the inverse function of the argument $\varphi(s)$ of such velocity vector. 
To evaluate this diameter we will estimate some quantities in the integral using the specific data in our setting, emphasizing which quantities change from $\theta \rightarrow \theta_{l}$ to $\theta \rightarrow \theta_{l-1}$ and which ones remain on both branches. From equations (77) and (16) we have $\varphi(s)=s^{2}\left[-\left|a_{2 k}\right|(k c)^{2} \sin \alpha_{2 k}+\mathcal{O}\left(s^{-1}\right)\right]$. Assuming $\sin \alpha_{2 k}<0$ (which does not affect the conclusions), it follows that $\psi(u)=u^{1 / 2}\left[C_{4}+\mathcal{O}\left(u^{-1 / 2}\right)\right]$ and $\psi^{\prime}(u)=u^{-1 / 2}\left[C_{5}+\mathcal{O}\left(u^{-1 / 2}\right)\right]$, where $C_{4}, C_{5}>0$ do not depend on the chosen branch. Thus,

$$
\begin{aligned}
r(\psi(u)) & =u^{a / 2}\left[C_{4}^{a} C_{1}+\mathcal{O}\left(u^{-1 / 2 k}\right)\right] e^{u^{1 / 2}\left[C_{2} C_{4}+\mathcal{O}\left(u^{-1 / 2 k}\right)\right]}, \\
h(u) & =u^{\frac{a-1}{2}}\left[C_{6}+\mathcal{O}\left(u^{-1 / 2 k}\right)\right] e^{u^{1 / 2}\left[C_{2} C_{4}+\mathcal{O}\left(u^{-1 / 2 k}\right)\right]} \\
& =u^{\frac{a-1}{2}}\left[C_{7}+\mathcal{O}\left(u^{-1 / 2 k}\right)\right] e^{C_{2} C_{4} u^{1 / 2}}
\end{aligned}
$$

with $C_{7}>0$. The key fact is that in the last expression, the constant $a$ exchanges from 1 to -1 when passing from one branch to the other, while the main coefficient in the exponent remains unchanged. For $n$ big enough, we can estimate $\Delta_{n}$ by

$$
2 \min _{u \in[n \pi,(n+1) \pi]} h(u+\alpha) \leq\left|\Delta_{n}\right| \leq 2 \max _{u \in[n \pi,(n+1) \pi]} h(u+\alpha),
$$

which thanks to the monotonicity of $h$, becomes

$$
\begin{aligned}
& 2(n \pi+\alpha)^{\frac{a-1}{2}}\left[C_{7}+\mathcal{O}\left(n^{-1 / 2 k}\right)\right] e^{C_{2} C_{4}(n \pi+\alpha)^{1 / 2}} \leq\left|\Delta_{n}\right| \\
\leq & 2((n+1) \pi+\alpha)^{\frac{a-1}{2}}\left[C_{7}+\mathcal{O}\left(n^{-1 / 2 k}\right)\right] e^{C_{2} C_{4}((n+1) \pi+\alpha)^{1 / 2}}
\end{aligned}
$$

and thus,

$$
\begin{aligned}
1 & \leq \frac{\left|\Delta_{n}\right|}{2(n \pi+\alpha)^{\frac{a-1}{2}}\left[C_{7}+\mathcal{O}\left(n^{-1 / 2 k}\right)\right] e^{C_{2} C_{4}(n \pi+\alpha)^{1 / 2}}} \\
& \left.\leq\left(\frac{(n+1) \pi+\alpha}{n \pi+\alpha}\right)^{\frac{a-1}{2}}\left[1+\mathcal{O}\left(n^{-1 / 2 k}\right)\right] e^{C_{1} C_{4}\left[((n+1) \pi+\alpha)^{1 / 2}-(n \pi+\alpha)^{1 / 2}\right.}\right] .
\end{aligned}
$$

The exponential term in the right-hand side of the last expression tends to one as $n \rightarrow \infty$, hence $\left|\Delta_{n}\right| \sim 2(n \pi+\alpha)^{\frac{a-1}{2}}\left[C_{7}+\mathcal{O}\left(n^{-1 / 2 k}\right)\right] e^{C_{2} C_{4}(n \pi+\alpha)^{1 / 2}}$. Now we compare this diameter at the two branches: as a passes from 1 to -1 while $C_{2} C_{4}$ do not change, we deduce that the diameters at both branches have a ratio of $n$ times a nonzero constant; also note that this ratio cannot come from a simple change in the $n$ variable (which is defined up to a constant), because $\Delta_{n+n_{0}}$ has the same growth as $\Delta_{n}$. In conclusion, there will be an interval between two consecutive maxima $\left[x_{\alpha}\left(s_{n}\right), x_{\alpha}\left(s_{n+2}\right)\right]$ of the faster spiral containing two maxima of the slower one, hence contradicting embeddedness. This finishes the proof of the proposition.

\section{Proof of Theorem 2}

Consider the height differential $\eta$ of our complete embedded minimal end $E \subset \mathbb{R}^{3}$. As $E$ is an annulus and has finite type, it can be parametrized conformally by a punctured neighborhood of infinity $\{|w| \geq r\}$ so that the puncture corresponds to $w=\infty$, and $\eta$ extends meromorphically through this puncture. Thus, we can write $\eta=h(w) d w$ for a holomorphic function $h$ on $\{|w| \geq r\}$ with at most a pole at $\infty$. As the third coordinate of the flux of $E$ along its boundary is zero, we deduce that the residue of $\eta$ at $\infty$ vanishes, hence there exists a holomorphic function $F$ on $\{|w| \geq r\}$ 
such that $d F=\eta$. Let us call $k$ to the order of pole $F$ at $\infty$ (thus $k-1$ is the order of pole $h$, which cannot be -1 because $\eta$ is residue-free). Moreover, choosing $r$ large enough we can insure that $F$ has a $k$-th holomorphic root on $\{|w| \geq r\}$ with a simple pole at $\infty$, i.e. $F(w)=(w \phi(w))^{k}$ for a holomorphic function $\phi$ on $\{|w| \geq r\} \cup\{\infty\}$ such that $\phi(\infty) \in \mathbb{C}-\{0\}$. Now in the new coordinate $z=w \phi(w)$, $|z| \geq R>0$, we express $\eta$ as $\eta=d\left((w \phi(w))^{k}\right)=k z^{k-1} d z$, or up to rescaling (because $k \neq 0$ ), $\eta=z^{k-1} d z$. On the other hand, as the stereographically projected Gauss map $g$ of $E$ is nonconstant and $\frac{d g}{g}$ extends meromorphically through the puncture, we conclude that $g$ has a finite number of zeros and poles in $\{|z| \geq R\}$. By enlarging $R$ we can assume without loss of generality that $g$ has no zeros or poles in $\{|z| \geq R\}$, which suffices for writing $g$ as $g(z)=z^{m} e^{H(z)}, m$ being an integer and $H$ a holomorphic function on $\{|z| \geq R\}$ that extends meromorphically to $\infty$ (a proof of this fact can be found in Theorem 1.1 of [10]). As $H$ has at most a pole at $\infty$, it can be written as in (2) for a certain integer $n$. In summary, we have parametrized $E$ by a complete minimal embedding $X: D(\infty, R) \longrightarrow \mathbb{R}^{3}$ with infinite total curvature and Weierstrass data $g(z)=z^{m} e^{H(z)}, \eta=z^{k-1} d z$.

Note that as $X$ has infinite total curvature, it follows that $g$ must have an essential singularity at $\infty$, or equivalently, that $n \geq 1$.

We want to reduce the problem to the case when $k=1$ and $X$ is proper. Then we can use Romon's proof 9] with the erratum given in Section 6, to show that the only possible Gauss map is of the form $g(z)=e^{a_{0}+a_{1} z+f(z)}$ where $a_{0} \in \mathbb{C}$, $a_{1}=i \nu \in i \mathbb{R}-\{0\}$ and $f$ is a holomorphic function that extends to $\infty$ with $f(\infty)=0$. Calling $\xi=\nu z-i a_{0}$ (thus we change the variable in the parameter domain by a translation and a real dilation, and $\xi$ is again defined in the exterior of a disk), we obtain that $g$ becomes $g(\xi)=e^{i \xi+f_{1}(\xi)}$ where $f_{1}(\xi)=f\left(\frac{1}{\nu}\left(\xi+i a_{0}\right)\right)$ (which is a well-defined holomorphic function in $|\xi| \geq R_{1}$ for $R_{1}$ large and satisfies $f_{1}(\infty)=0$ ), while $\eta=\frac{1}{\nu} d \xi$, and Theorem 2 follows. Hence, our Theorem 2 reduces to check that from the general case ( $X$ is complete, $k \neq 0$ integer, $n \geq 1$ ) we arrive to Romon's setting ( $X$ is proper, $k=1, n \geq 1$ ). As the proof is rather long, we will divide it in several steps.

Step 1: Reduction to the case $n$ is a positive multiple of $k \geq 1$. If $k \leq-1$, the surface cannot be complete because one can compute the intrinsic length of a proper arc $\Gamma \subset|g|^{-1}(1) \subset D(\infty, R)$ (the essential singularity of $g$ at infinity insures the existence of such an arc) as $\int_{\Gamma} d s=\frac{1}{2} \int_{\Gamma}\left(|g|^{-1}+|g|\right)|\eta|=\int_{\Gamma}|z|^{k-1}|d z|$. We saw in Section 3 that $\Gamma$ can be parametrized by $\Gamma(r)=r e^{i \theta(r)}$, where $\theta(r)$ is defined by equation (12) (which does not depend on any assumption on $k$ ), hence with the notation of that section we have $\cos \left(n \theta(r)+\alpha_{n}\right)=-\frac{m \log r}{\left|a_{n}\right| r^{n}}+\mathcal{O}\left(r^{-1}\right)$, which by derivation gives $\theta^{\prime}(r)=\mathcal{O}\left(r^{-n}\right)+\mathcal{O}\left(r^{-2}\right)=\mathcal{O}\left(r^{-1}\right)$. As $\left|\Gamma^{\prime}(r)\right|=\left|1+i r \theta^{\prime}(r)\right|$ and $\int_{r_{0}}^{\infty} r^{k-1} d r$ converges when $k \leq-1$, we conclude that the length of $\Gamma$ is finite, a contradiction.

Now we can assume $n, k \geq 1$. By Proposition $1, n$ must be a multiple of $k$, and our first step is finished.

Step 2: The case $n=k \geq 1$. Now $H(z)=a_{k} z^{k}+\ldots+a_{1} z+a_{0}+f(z)$, where $f$ is a holomorphic function in $D(\infty, R) \cup\{\infty\}$ with $f(\infty)=0$.

Claim 3. $X$ is a proper embedding and has bounded Gaussian curvature. 
Proof of Claim 3. The expression of the absolute Gaussian curvature in terms of our Weierstrass data is

$$
|K|=\frac{4}{\left(|g|+|g|^{-1}\right)^{4}} \frac{|d g / g|^{2}}{|\eta|^{2}}=\frac{1}{4(\cosh A(z))^{4}} \frac{\left|\frac{m}{z}+H^{\prime}(z)\right|^{2}}{r^{2(k-1)}} \leq \frac{\left|\frac{m}{z}+H^{\prime}(z)\right|^{2}}{4 r^{2(k-1)}}
$$

where $z=r e^{i \theta}$. As $n=k$, it follows that $\left|\frac{m}{z}+H^{\prime}(z)\right|^{2}$ grows as $r^{2(k-1)}$ times a constant when $r \rightarrow \infty$, thereby proving the boundness of $K$. As $X(D(\infty, R))$ is a complete embedded minimal annulus with compact boundary and bounded Gaussian curvature, a theorem of Meeks and Rosenberg [6] insures that $X$ is proper (indeed, they even prove that $X(D(\infty, R))$ has an embedded regular neighborhood of fixed radius. 6] studies complete embedded minimal surfaces of bounded curvature and empty boundary, but their result extends to the case of compact boundary). Now our claim is proved.

We now continue with the proof of Step 2. If $k=1$, then [9] together with Section 6 imply that our surface has the desired Weierstrass data, as we remarked before starting Step 1.

Suppose from now on that $n=k \geq 2$. Using Proposition 1 and Remark 1 we conclude that $a_{k} \in i \mathbb{R}$ and $a_{1}=\ldots=a_{k-1}=0$. We will prove that this case cannot occur by finding a level curve $\gamma_{c, l_{1}}$ whose image by $X$ cuts to a quasivertical axis contained in an upper leaf $X\left(S_{l_{2}}\right)$ with $l_{1} \neq l_{2}$, which will force $X$ to be nonembedded. Consider $c>0$ large and $l \in\{1, \ldots, 2 k-2\}$ odd. First, recall from Proposition 2 that the horizontal level curve $X\left(\gamma_{c, l}\right) \subset X\left(S_{l}\right)$ has two divergent branches whose arguments converge to the arguments of two halflines starting from the origin in $\left\{x_{3}=c\right\}$, and these halflines form an angle which does not depend on the height $c$ but they rotate around the $x_{3}$-axis as $c$ increases. As $X$ is a proper embedding, $X\left(\gamma_{c, l}\right)$ separates $\left\{x_{3}=c\right\}$ in two components that we denote by $\mathcal{C}_{c}^{+}, \mathcal{C}_{c}^{-}$, so that $\mathcal{C}_{c}^{+}$is the one where the horizontal projection of the Gauss map of $X(D(\infty, R))$ points to. On the other hand, Proposition 3 applied to the upper leaves $X\left(S_{l}\right), X\left(S_{l+2}\right)$ give two quasivertical axes $\zeta_{1}=X\left(\Gamma_{1}\right) \subset X\left(S_{l}\right)$, $\zeta_{2}=X\left(\Gamma_{2}\right) \subset X\left(S_{l+2}\right)$, where $\Gamma_{1}, \Gamma_{2} \subset D(\infty, R)$ are divergent curves respectively asymptotic to the rays of constant argument $\Theta_{j_{1}}, \Theta_{j_{2}}$ with $\cos \left(k \Theta_{j}+\alpha_{k}\right)=0$, $j=j_{1}, j_{2}$ (as $n=k$ and $a_{k} \in i \mathbb{R}$, the angle $\Theta_{j_{1}}$ (resp. $\Theta_{j_{2}}$ ) bisects the sector $S_{l}$ (resp. $\left.S_{l+2}\right)$ ). By definition, each $\zeta_{j}$ is asymptotic to a vertical halfline $t_{j} \subset\left\{x_{3} \geq\right.$ $0\}$ with boundary at height zero. As $X(D(\infty, R))$ admits an embedded regular neighborhood of constant radius (see the proof of Claim 3) and $\zeta_{1}, \zeta_{2}$ lie in different upper leaves, the vertical halflines $t_{1}$ and $t_{2}$ must also be distinct. By definition of quasivertical axis, for $c$ large $X\left(\gamma_{c, l}\right)$ passes through a point extremely close to $t_{1} \cap\left\{x_{3}=c\right\}$ and the same holds with $X\left(\gamma_{c, l+2}\right)$ and $t_{2} \cap\left\{x_{3}=c\right\}$. Let us call $I_{c} \subset \mathbb{R}$ to the interval of arguments covered by the velocity vector of the planar curve $X\left(\gamma_{c, l}\right)$.

Claim 4. In the above situation, the length of $I_{c}$ cannot diverge to $+\infty$ as $c$ increases.

Proof of Claim 4. Equation (6) gives that length $\left(I_{c}\right)=\operatorname{length}\left(B(] \theta_{l-1}, \theta_{l}[)\right)$. Moreover, equation (3) and our assumptions $a_{k} \in i \mathbb{R}$ and $a_{1}=\ldots=a_{k-1}=0$ give

$$
B(\theta)=\left[m \theta+\operatorname{Imag}\left(a_{0}\right)+\left|a_{k}\right| k c \sin \alpha_{k}\right]+\mathcal{O}\left(\left(\frac{c}{\cos k \theta}\right)^{-1 / k}\right) .
$$


As a function of $\theta$, the linear expression enclosed in brackets has an image interval of length $|m|\left(\theta_{l}-\theta_{l-1}\right)=\frac{|m|}{k} \pi$. This fact jointly with the inequality $\left|\mathcal{O}\left(\left(\frac{c}{\cos k \theta}\right)^{-1 / k}\right)\right|$ $\leq C_{0} c^{-1 / k}$ for a positive constant $C_{0}$, imply that

$$
\operatorname{length}\left(B(] \theta_{l-1}, \theta_{l}[)\right) \leq \frac{|m|}{k} \pi+2 C_{0} c^{-1 / k},
$$

which is bounded as $c \rightarrow \infty$. Now Claim 4 is proved.

Take $c_{0}>0$ large and denote by

$$
\begin{aligned}
& \mathcal{A}^{+}=\left\{c>c_{0}: t_{2} \cap\left\{x_{3}=c\right\} \subset \mathcal{C}_{c}^{+}\right\}, \\
& \mathcal{A}^{-}=\left\{c>c_{0}: t_{2} \cap\left\{x_{3}=c\right\} \subset \mathcal{C}_{c}^{-}\right\}, \\
& \mathcal{A}^{0}=\left\{c>c_{0}: t_{2} \cap\left\{x_{3}=c\right\} \subset X\left(\gamma_{c, l}\right)\right\} .
\end{aligned}
$$

Obviously, $\left\{c>c_{0}\right\}$ is the disjoint union of $\mathcal{A}^{+}, \mathcal{A}^{-}$and $\mathcal{A}^{0}$. Moreover, each connected component of any of these three sets is bounded: for $\mathcal{A}^{+}$and $\mathcal{A}^{-}$this is a direct consequence of Claim 4. For $\mathcal{A}^{0}$, we argue as follows. Recall that the quasivertical axis $\zeta_{2}$ projects vertically into a spiral that converges to the point $t_{2} \cap\left\{x_{3}=0\right\}$. As $X$ is embedded, $X\left(\gamma_{c, l}\right)$ must be disjoint of $\zeta_{2}$ for any $c$, hence if $c \in \mathcal{A}^{0}$ for all $c$ greater than a positive number $c_{1}$, then when $c$ increases the curve $X\left(\gamma_{c, l}\right)$ must rotate locally around $t_{2} \cap\left\{x_{3}=c\right\}$ infinitely many times in order to avoid $\zeta_{2}$. As $X\left(\gamma_{c, l}\right)$ passes through a point extremely close to $t_{1} \cap\left\{x_{3}=c\right\}$ for all $c$ large enough, this implies that the length of $I_{c}$ goes to $+\infty$ as $c$ increases, in contradiction with Claim 4.

These properties imply that $t_{2}$ must cut to $\mathcal{C}_{c}^{+}$and to $\mathcal{C}_{c}^{-}$infinitely often as $c$ increases. As $\zeta_{2}$ is asymptotic to $t_{2}$, it follows that $\zeta_{2}=X\left(\Gamma_{2}\right)$ also cuts to $\mathcal{C}_{c}^{+}$ and to $\mathcal{C}_{c}^{-}$infinitely often, hence it must intersect to $X\left(\gamma_{c, l}\right)$, which is the desired contradiction. This finishes the proof of Step 2.

Step 3: The case $n=p k, p \geq 2$. Again the argument consists of finding two disjoint divergent curves $\beta_{1}, \beta_{2} \subset D(\infty, R)$ whose image by $X$ intersect each other. These image curves $X\left(\beta_{i}\right)$ will roll up infinitely often on a vertical cylinder in opposite directions and their third coordinate will be proper, properties that guarantee the desired intersections.

As $p \geq 2$, Proposition $3 i$ ) assures that each leaf contains at least $p-1$ quasivertical axes and there exists an integer $\mu, 0 \leq \mu \leq 2 k$ which counts how many nonproper curves there are in $X\left(|g|^{-1}\left(e^{A}\right)\right)$. We assert that there exists a leaf $X\left(S_{l}\right)$ containing at least two quasivertical axes. If $p \geq 3$, this is clear from the previous application of Proposition $3 i$ ). If $p=2$, then the embeddedness of $X$ together with Proposition 5 show that $\mu<2 k$, and therefore, Proposition $3 i$ ) again insures that there exists a leaf $X\left(S_{l}\right)$ containing at least two quasivertical axes, thereby proving our assertion.

Without loss of generality, we can suppose that the leaf $X\left(S_{l}\right)$ that contains two quasivertical axes is an upper leaf. By Proposition $4, X\left(S_{l}\right)$ has at least $p-1$ oblique rays, hence there exist divergent curves $\Gamma_{1}, \Gamma_{2}, \widehat{\Gamma}$ contained in the sector $S_{l} \subset D(\infty, R)$ such that $|g|$ is constantly one along $\Gamma_{1}, \Gamma_{2}$ while $\arg (g)$ is constantly zero along $\widehat{\Gamma}$ (or equivalently, $\left.A\right|_{\Gamma_{1}}=\left.A\right|_{\Gamma_{2}}=\left.B\right|_{\widehat{\Gamma}}=0$ ), and $X\left(\Gamma_{1}\right), X\left(\Gamma_{2}\right)$ are quasivertical axes asymptotic to vertical halflines $t_{1}, t_{2} \subset\left\{x_{3} \geq 0\right\}$ while $X(\widehat{\Gamma})$ is an oblique ray. Moreover, it is clear that we can choose these curves so that they are cyclicly consecutive in $\{|z| \geq R\}$, i.e. $\Gamma_{1}, \widehat{\Gamma}, \Gamma_{2}$ are respectively asymptotic 


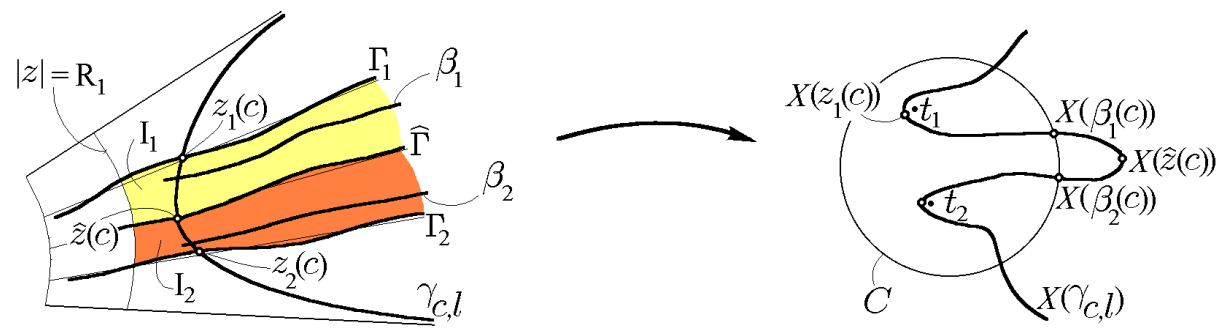

FIGURE 1.

to the rays of angles $\Theta_{j}+\frac{\pi}{n}, \Theta_{j}+\frac{\pi}{2 n}, \Theta_{j}$ for a certain $j \in\{1, \ldots, 2 n\}$ (with the same notation as in Section (3). In particular, the limit angle $\Theta_{j}+\frac{\pi}{2 n}$ of $\widehat{\Gamma}$ satisfies $\cos k\left(\Theta_{j}+\frac{\pi}{2 n}\right) \neq 0$, thus $\left.x_{3}\right|_{\hat{\Gamma}}$ diverges monotonically to $+\infty$; see Section 3 Working on a proper subannulus $\left\{|z| \geq R_{1}\right\}$ with $R_{1}$ large, the above curves joint with $\left\{|z|=R_{1}\right\} \cap S_{l}$ determine two regions $I_{1}, I_{2}$ where $B$ has opposite signs, namely, we define $I_{1}$ to be the proper region in $S_{l}$ delimited by $\Gamma_{1}, \widehat{\Gamma}$ and $\left\{|z|=R_{1}\right\}$, while $I_{2}$ is the proper region in $S_{l}$ bounded by $\widehat{\Gamma}, \Gamma_{2}$ and $\left\{|z|=R_{1}\right\}$; see Figure 1

As $B$ is zero along $\widehat{\Gamma}$ and the three above curves are consecutive, $B$ has constant sign on $I_{1}$ (say positive) and opposite sign on $I_{2}$. Consider a vertical cylinder $C$ in $\left\{x_{3} \geq 0\right\} \subset \mathbb{R}^{3}$ of radius big enough so that $t_{1}, t_{2}$ are contained in the simply connected component $U$ of $\left\{x_{3} \geq 0\right\}-C$. Take $c>0$ large and consider the horizontal level curve $\gamma_{c, l} \subset S_{l}$. For $i=1,2, \gamma_{c, l}$ crosses the region $I_{i}$ intersecting $\Gamma_{i}$ at a point $z_{i}(c)$ and $\widehat{\Gamma}$ at a point $\widehat{z}(c)$. The image through $X$ of the intersection point $z_{i}(c)$ is on the quasivertical axis $X\left(\Gamma_{i}\right)$, hence it belongs to $U$. On the other hand, as $X(\widehat{\Gamma})$ is an oblique ray, the squared length of its horizontal projection is proper, thus $X(\widehat{z}(c))$ is in $\left\{x_{3} \geq 0\right\}-\operatorname{closure}(U)$. Therefore, by continuity $\gamma_{c, l}$ must intersect $X^{-1}\left(C \cap\left\{x_{3}=c\right\}\right)$ along the open connected subarc between $z_{1}(c)$ and $\widehat{z}(c)$ in a point $\beta_{1}(c)$ and between $\widehat{z}(c)$ and $z_{2}(c)$ in a point $\beta_{2}(c)$. These intersections allow us to consider two divergent curves $\beta_{1} \subset I_{1}, \beta_{2} \subset I_{2}$ parametrized by the height $c$, so that $X\left(\beta_{i}(c)\right) \in C \cap\left\{x_{3}=c\right\}$ for any $c>0$ large (the curves $\beta_{i}(c)$ diverge in $I_{i}$ as $c \rightarrow+\infty$ because the horizontal level curves $\left\{\gamma_{c, l}\right\}_{c>0}$ foliate $\left.I_{i}\right)$. The argument will be complete if we prove that $B \circ \beta_{1}$ (resp. $B \circ \beta_{2}$ ) diverges to $+\infty$ (resp. $-\infty$ ) as $c \rightarrow+\infty$. If on the contrary, $B \circ \beta_{1}$ remains bounded for any $c$ large, then the principal term of the expansion of $B$ in equation (3) implies that the argument of $\beta_{1}$ must converge to one of the zeros of the equation $\sin \left(n \theta+\alpha_{n}\right)=0$, or in other words, $\beta_{1}$ must be asymptotic to $\widehat{\Gamma}$. But in this case, the principal term of the expansion of $A$ in (3) grows as $|z|^{n}$ times a nonzero constant, hence by (4) the length of $\frac{d}{d c}\left(\left(x_{1}+i x_{2}\right)\left(\beta_{1}\right)\right)$ grows exponentially while its argument remains bounded. A similar argument as the one in Lemma 2 implies that $\left(x_{1}+i x_{2}\right)\left(\beta_{1}\right)$ is a divergent planar curve, hence it cannot be contained in the circle obtained by the projection of $C$ into a horizontal plane, a contradiction. Now as $B$ has positive sign on $I_{1}, B \circ \beta_{1}$ must diverge to $+\infty$ as $c \rightarrow \infty$. An analogous argument holds with $B \circ \beta_{2}$ (note that $B$ is negative everywhere on $I_{2}$ ), and the proof of Step 3 is complete. 


\section{Proof of Theorem 3}

Consider a pair $\left(g(z)=e^{i z+f(z)}, \eta=d z\right)$ defined on $\{|z| \geq R\}$, where $R>0$ and $f$ is a holomorphic function in $\{|z| \geq R\} \cup\{\infty\}$ with $f(\infty)=0$. As we want $(g, \eta)$ to produce a well-defined end, we need the real period along the boundary curve $\{|z|=R\}$ to be zero. Note that this condition holds immediately when the end is part of a properly embedded minimal surface $M$ with finite topology, because in that case Collin's Theorem [1] insures that $M$ has only one end, and thus, the curve $\{|z|=R\}$ bounds a compact domain in $M$ and the Divergence Theorem guarantees that the period is zero (in fact, the same argument proves that the flux along $\{|z|=R\}$ also vanishes). The period condition for an isolated end, which only involves the two first components because $\eta$ is exact, is

$$
\overline{\int_{\{|z|=R\}} e^{-i z-f(z) d z}}=\int_{\{|z|=R\}} e^{i z+f(z)} d z .
$$

We assume from now on that the pair $(g, \eta)$ defined on $D(\infty, R)$ fulfills the above period condition. As a consequence, it defines a complete unbranched minimal immersion $X(z)=\frac{1}{2}$ Real $\int^{z}\left(g^{-1}-g, i\left(g^{-1}+g\right), 2\right) \eta$ with bounded Gaussian curvature $K$ (the induced metric is $d s^{2}=\lambda^{2}|d z|^{2}$ where $\lambda=\frac{1}{2}\left(|g|+|g|^{-1}\right.$ ), hence the inequality $\lambda \geq 1 / 2$ gives that $d s^{2}$ is unbranched and complete; for the boundness of $K$, use the estimate in the proof of Claim 3). Note that the third coordinate of the immersion is $x_{3}(z)=\operatorname{Real}(z)$, thus the horizontal level set at height $c \in \mathbb{R}$ corresponds in the parameter domain with the vertical line $D(\infty, R) \cap\{\operatorname{Real}(z)=c\}$. Moreover, Proposition 2 insures that every horizontal level curve has two divergent branches in $\left\{x_{3}=c\right\}$ whose arguments converge to the arguments of two halflines starting from the origin in that plane. In particular, if $\left\{z_{n}\right\}_{n} \subset D(\infty, R)$ is any sequence with $\left|z_{n}\right| \rightarrow \infty$, then either $\left\{\operatorname{Real}\left(z_{n}\right)\right\} \rightarrow \infty$ and in this case $x_{3}\left(z_{n}\right)$ is unbounded, or $\left\{\operatorname{Real}\left(z_{n}\right)\right\}_{n}$ converges but $\left\{\operatorname{Imag}\left(z_{n}\right)\right\}$ diverges, in which case Proposition 2 shows that $\left\{X\left(z_{n}\right)\right\}_{n}$ diverges in bounded height. This proves that our immersion $X$ is proper.

The end is embedded. We now prove that a representative of the end is embedded by showing that there exists $R_{1} \geq R$ such that every horizontal section of $X\left(D\left(\infty, R_{1}\right)\right)$ is embedded. The argument that follows is inspired in beautiful ideas in [4]. Given $c \in \mathbb{R}$, the horizontal level curve at height $c$ is parametrized in the parameter domain by the curve $\gamma_{c}(v)=c+i v$, which has one or two connected components depending on the cases $|c| \geq R$ or $|c|<R$, respectively. As the unit tangent vector along $X\left(\gamma_{c}\right)$ can be chosen as $i \frac{g}{|g|}$, the second derivative of $X\left(\gamma_{c}\right)$ with respect to its arc length $s$ is $\frac{d^{2} X\left(\gamma_{c}\right)}{d s^{2}}=\frac{d}{d s}\left(i \frac{g}{|g|}\right)=\frac{d}{d z}\left(i \frac{g}{|g|}\right) \frac{d \gamma_{c}}{d s}=\frac{i}{2} \frac{g^{\prime}}{|g|} \frac{d \gamma_{c}}{d s}$, thus the planar curvature of $X\left(\gamma_{c}\right)$ is given by $\kappa=\left\langle\frac{d^{2} X\left(\gamma_{c}\right)}{d s^{2}}, \frac{g}{|g|}\right\rangle=\frac{-1}{2} \operatorname{Imag}\left(\frac{g^{\prime}}{g} \frac{d \gamma_{c}}{d s}\right)$. As $d s=\lambda|d z|$, we have along $\gamma_{c}$ that $\lambda^{-1}=\left|\frac{d z}{d s}\right|=\left|\frac{d \gamma_{c}}{d s}\right|$, hence $\frac{d \gamma_{c}}{d s}=i \lambda^{-1}$ and the curvature of $X\left(\gamma_{c}\right)$ becomes $\kappa=-\frac{1}{2 \lambda} \operatorname{Real}\left(\frac{g^{\prime}}{g}\right)=-\frac{1}{2 \lambda} \operatorname{Real}\left(i+f^{\prime}\right)=-\frac{1}{2 \lambda} \operatorname{Real}\left(f^{\prime}\right)$.

On the other hand, as $f$ is holomorphic and $f(\infty)=0$, the expansion of $f$ has only negative powers of $z$, so we can find $C>0$ depending only on $f$ such that

$$
|f(z)| \leq C|z|^{-1}, \quad\left|f^{\prime}(z)\right| \leq C|z|^{-2} \quad \text { for any } z \in D(\infty, R) .
$$


Assume that $|c| \geq R$. Thus, $\gamma_{c}(v)=c+i v$ is connected and $v$ runs along the whole real line. The absolute total curvature of $X\left(\gamma_{c}\right)$ is $\int_{\gamma_{c}}|\kappa(s)| d s=\int_{-\infty}^{\infty}|\kappa(v)| \lambda d v=$ $\frac{1}{2} \int_{-\infty}^{\infty}\left|\operatorname{Real}\left(f^{\prime}\left(\gamma_{c}\right)\right)\right| d v$. Using the estimate of $\left|f^{\prime}\right|$ in (19), it follows that

$$
\int_{\gamma_{c}}|\kappa(s)| d s \leq \frac{C}{2} \int_{-\infty}^{\infty} \frac{d v}{c^{2}+v^{2}}=\frac{C \pi}{2|c|} .
$$

Therefore, the absolute total curvature of $X\left(\gamma_{c}\right)$ is strictly less than $\pi$ provided that $|c|>\frac{C}{2}$, and we have proved that

- If $|c|>\max \left(R, \frac{C}{2}\right)$, then $X(D(\infty, R)) \cap\left\{x_{3}=c\right\}$ is an embedded curve.

Now consider any height $c$ (although we are interested in the embeddedness of the horizontal sections in the range $\left.|c| \leq \max \left(R, \frac{C}{2}\right)\right)$. Take a fixed $v_{0}>R$, to be defined later. Hence, the (possibly nonconnected) level set $\gamma_{c}$ is defined in $|v| \geq v_{0}$. As before,

$$
\int_{v_{0}}^{\infty}|\kappa(s)| d s \leq \frac{C}{2} \int_{v_{0}}^{\infty} \frac{d v}{c^{2}+v^{2}} \leq \frac{C}{2} \int_{v_{0}}^{\infty} \frac{d v}{v^{2}}=\frac{C}{2 v_{0}},
$$

and similarly $\int_{-\infty}^{-v_{0}}|\kappa(s)| d s \leq \frac{C}{2 v_{0}}$. Thus, both absolute total curvatures of $\left.X\left(\gamma_{c}\right)\right|_{\left\{v \geq v_{0}\right\}}$ and $\left.X\left(\gamma_{c}\right)\right|_{\left\{v \leq-v_{0}\right\}}$ are strictly less than $\pi$ if we take $v_{0}>\frac{C}{2 \pi}$ (note that $v_{0}$ does not depend on $\left.c\right)$, and we deduce that

- If $\left|v_{0}\right|>\max \left(R, \frac{C}{2 \pi}\right)$, then $\left.X\left(\gamma_{c}\right)\right|_{\left\{v \geq v_{0}\right\}}$ and $\left.X\left(\gamma_{c}\right)\right|_{\left\{v \leq-v_{0}\right\}}$ are separately embedded.

Thus, by suitably enlarging $R$ we can assume that $X: D(\infty, R) \longrightarrow \mathbb{R}^{3}$ satisfies that $X\left(\gamma_{c}\right)$ is embedded and connected for $|c| \geq R$, while for $|c|<R$ the two connected components $\gamma_{c}^{+}, \gamma_{c}^{-}$of $\gamma_{c}$ are separately embedded (we label these components so that $\gamma_{c}^{+} \subset\{\operatorname{Imag}(z)>0\}$ and $\left.\gamma_{c}^{-} \subset\{\operatorname{Imag}(z)<0\}\right)$. We claim that there exists $v_{0} \geq R$ such that $\left.\left.X\left(\gamma_{c}^{+}\right)\right|_{\left\{v \geq v_{0}\right\}} \cap X\left(\gamma_{c}^{-}\right)\right|_{\left\{v \leq-v_{0}\right\}}=\varnothing$ for all $c$ with $|c|<R$. Consider the set

$$
\mathcal{A}=\{c+i v:|c| \leq R \text { and there exists } w \neq v \text { with } X(c+i v)=X(c+i w)\} .
$$

Clearly, it suffices to prove that there exists $v_{0} \geq R$ independent of $c$ such that if $|c| \leq R$ and $|v| \geq v_{0}$, then $c+i v \notin \mathcal{A}$, or in other words, we only need to show that the map $c+i v \mapsto|v|$ is bounded on $\mathcal{A}$. Reasoning by contradiction, suppose that there exists a sequence $\left\{c_{n}+i v_{n}\right\}_{n} \subset \mathcal{A}$ with $\left|v_{n}\right| \rightarrow+\infty$. By definition, for each $n$ we can find $w_{n} \neq v_{n}$ such that $X\left(c_{n}+i v_{n}\right)=X\left(c_{n}+i w_{n}\right)$. As $\left|c_{n}\right| \leq R$, it follows that $\left\{c_{n}\right\}_{n}$ converges to $c_{\infty} \in[-R, R]$, after passing to a subsequence. We can also assume without loss of generality that $v_{n}>0$ for each $n$. As $\gamma_{c}^{+}, \gamma_{c}^{-}$are separately embedded when $|c|<R$, it follows that $w_{n}<0$ for all $n$. Moreover, $w_{n}$ diverges to $-\infty$ (otherwise, $\left\{w_{n}\right\}_{n}$ can be supposed to converge to $w_{\infty}<0$ after taking a subsequence, and then $X\left(c_{n}+i v_{n}\right)=X\left(c_{n}+i w_{n}\right) \rightarrow X\left(c_{\infty}+i w_{\infty}\right) \in \mathbb{R}^{3}$, which contradicts that $X\left(\gamma_{c}^{+}\right)$is a divergent arc by Proposition 2). Therefore, equation (6) gives that the tangent vector to $X\left(\gamma_{c}^{+}\right)$(resp. $X\left(\gamma_{c}^{-}\right)$) has an argument converging to $B\left(\frac{\pi}{2}\right)-\frac{\pi}{2}$ (resp. $\left.B\left(\frac{-\pi}{2}\right)-\frac{\pi}{2}\right)$, where $g=e^{A+i B}$ as in former sections. In our case, these limit arguments are respectively $c-\frac{\pi}{2}, c+\frac{\pi}{2}$ (we can use equation (11) in order to compute them), which contradicts that $X\left(c_{n}+i v_{n}\right)=X\left(c_{n}+i w_{n}\right)$ for all $n$. This contradiction shows that $c+i v \mapsto|v|$ is bounded on $\mathcal{A}$, finishing the proof of the embeddedness of $X\left(D\left(\infty, R_{1}\right)\right)$ for $R_{1}$ large enough. 


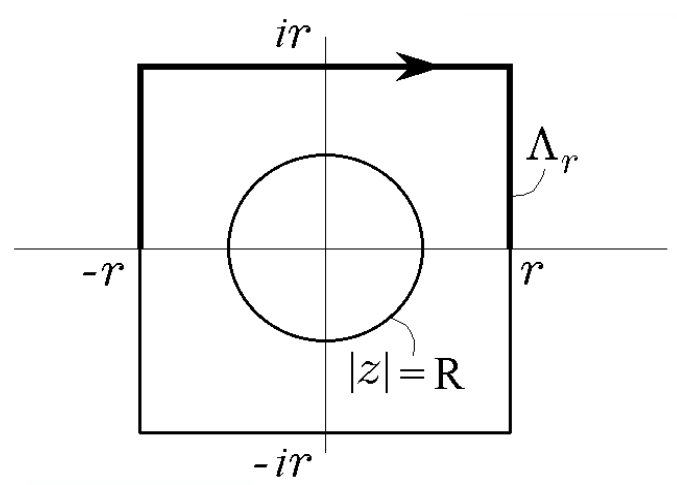

FIGURE 2.

The end is $C^{0}$-asymptotic to the end of a Helicoid. Recall from Section 3 that $|g|^{-1}(1)$ consists of two divergent curves $\Gamma_{1}, \Gamma_{2}$ which are asymptotic to the components of $D(\infty, R) \cap\{\operatorname{Imag}(z)=0\}$ and have $x_{3}\left(\Gamma_{j}\right)$ unbounded. We label these curves so that $\Gamma_{1}$ (resp. $\Gamma_{2}$ ) is asymptotic to the negative (resp. positive) real axis, hence $X\left(\Gamma_{1}\right) \subset\left\{x_{3}<0\right\}$ (resp. $X\left(\Gamma_{2}\right) \subset\left\{x_{3}>0\right\}$ ). Equivalently, the limit arguments of $\Gamma_{1}, \Gamma_{2}$ are respectively $\Theta_{1}=\pi, \Theta_{2}=0$ with the notation of Section 3 Using case ii) of Proposition 3 we deduce that these two curves are applied by $X$ on two quasivertical axes in both halfspaces of $\mathbb{R}^{3}-\left\{x_{3}=0\right\}$.

We now prove that if the flux of $X$ along the boundary curve $\{|z|=R\}$ vanishes, then the asymptotic vertical halflines $t_{1}, t_{2}$ to these quasivertical axes project vertically onto the same point in the plane $\left\{x_{3}=0\right\}$. With this aim, take $r>R$ and consider the boundary $\partial S_{r}$ of the square $S_{r}$ with vertices $r+i r, r-i r,-r-i r,-r+$ ir. $\partial S_{r}$ can be written as $\Lambda_{r}-\overline{\Lambda_{r}}$, where $\Lambda_{r}$ is the polygonal arc with vertices $-r,-r+i r, r+i r, r$ (the orientations on $\partial S_{r}$ and $\Lambda_{r}$ are chosen so that both lists of vertices are naturally well-ordered; see Figure 22). As $\{|z|=R\}$ and $\partial S_{r}$ bound a compact domain in the surface, it follows that the flux of $X$ along $\partial S_{r}$ equals zero. This is equivalent to the following two equalities (recall that the height differential $\eta$ is exact):

$$
\int_{\Lambda_{r}} g d z=\int_{\overline{\Lambda_{r}}} g d z, \quad \int_{\Lambda_{r}} g^{-1} d z=\int_{\overline{\Lambda_{r}}} g^{-1} d z .
$$

As the quasivertical axes are asymptotic to the components of the real axis in $D(\infty, R)$, in order to prove that the vertical halflines $t_{1}, t_{2}$ project onto the same point in $\left\{x_{3}=0\right\}$ it remains to check that $\left(x_{1}+i x_{2}\right)(r)-\left(x_{1}+i x_{2}\right)(-r)$ tends to zero as $r \rightarrow+\infty$. Using (44), this horizontal distance can be written as

$$
\begin{aligned}
\left(x_{1}+i x_{2}\right)(r)-\left(x_{1}+i x_{2}\right)(-r) & =\int_{\Lambda_{r}} d\left(x_{1}+i x_{2}\right)=\frac{1}{2} \int_{\Lambda_{r}}\left(\overline{\frac{1}{g} \frac{d \Lambda_{r}}{d s}}-g \frac{d \Lambda_{r}}{d s}\right) d s \\
& =\frac{1}{2}\left(\int_{\Lambda_{r}} \bar{g}^{-1} d \bar{z}-\int_{\Lambda_{r}} g d z\right),
\end{aligned}
$$

where we have called $z=\Lambda_{r}(s)$. Equation (20) implies that the last expression equals $\frac{1}{2}\left(I_{2}-I_{1}\right)$, where $I_{1}=\int_{\Lambda_{r}} g d z$ and $I_{2}=\int_{\Lambda_{r}} \bar{g}^{-1} d \bar{z}$. We now compute 
separately these two integrals:

$$
\begin{aligned}
I_{1} & =\int_{0}^{r} g(-r+i v) i d v+\int_{-r}^{r} g(u+i r) d u-\int_{0}^{r} g(r+i v) i d v \\
& =e^{-r} \int_{-r}^{r} e^{i u+f(u+i r)} d u+i \int_{0}^{r} e^{-v}\left(e^{-i r+f(-r+i v)}-e^{i r+f(r+i v)}\right) d v .
\end{aligned}
$$

If we take $r$ such that $\frac{r}{2 \pi}$ is an integer, $I_{1}$ becomes

$$
I_{1}=e^{-r} \int_{-r}^{r} e^{i u+f(u+i r)} d u+i \int_{0}^{r} e^{-v}\left(e^{f(-r+i v)}-e^{f(r+i v)}\right) d v,
$$

and a similar computation for $I_{2}$ gives

$$
I_{2}=e^{-r} \int_{-r}^{r} e^{i u-\overline{f(u-i r)}} d u+i \int_{0}^{r} e^{-v}\left(e^{-\overline{f(-r-i v)}}-e^{-\overline{f(r-i v)}}\right) d v .
$$

Thus, both $I_{1}$ and $I_{2}$ are the sum of two integrals and each one of these four integrals can be estimated using (19) as follows:

$$
\begin{aligned}
\left|e^{-r} \int_{-r}^{r} e^{i u+f(u+i r)} d u\right| & \leq e^{-r} \int_{-r}^{r} e^{\operatorname{Real} f(u+i r)} d u \\
& \leq e^{-r} \int_{-r}^{r} e^{C|u+i r|^{-1}} d u \\
& \leq e^{-r} \int_{-r}^{r} e^{C r^{-1}} d u=2 r e^{-r+C r^{-1}},
\end{aligned}
$$

and analogously, $\left|e^{-r} \int_{-r}^{r} e^{i u-\overline{f(u-i r)}} d u\right| \leq 2 r e^{-r+C r^{-1}}$, while

$$
\left|i \int_{0}^{r} e^{-v}\left(e^{f(-r+i v)}-e^{f(r+i v)}\right) d v\right| \leq \int_{0}^{r} e^{-v}\left|e^{f(-r+i v)}-e^{f(r+i v)}\right| d v .
$$

Using (19), it is straightforward to check that both $e^{f(-r+i v)}$ and $e^{f(r+i v)}$ can be expressed as $1+\mathcal{O}\left(r^{-1}\right)$, hence the last expression is of the type $\int_{0}^{r} e^{-v} \mathcal{O}\left(r^{-1}\right) d v \leq C_{1} r^{-1}\left(1-e^{-r}\right)$, for a constant $C_{1}>0$ independent of $r$. Similarly, $\left|i \int_{0}^{r} e^{-v}\left(e^{-\overline{f(-r-i v)}}-e^{-\overline{f(r-i v)}}\right) d v\right| \leq C_{1} r^{-1}\left(1-e^{-r}\right)$. In summary,

$\left|\left(x_{1}+i x_{2}\right)(r)-\left(x_{i}+i x_{2}\right)(-r)\right| \leq \frac{1}{2}\left(\left|I_{2}\right|+\left|I_{1}\right|\right) \leq 2 r e^{-r+C r^{-1}}+C_{1} r^{-1}\left(1-e^{-r}\right)$,

which tends to zero as $r \rightarrow+\infty$. Consequently, the vertical halflines $t_{1}, t_{2}$ asymptotic to the quasivertical axes $\Gamma_{1}, \Gamma_{2}$ are part of the same vertical straightline, which will be supposed to be the $x_{3}$-axis after a suitable translation.

Finally, consider the vertical Helicoid $H \subset \mathbb{R}^{3}$ with Weierstrass data $(g(z)=$ $\left.e^{i z}, \eta=d z\right), z \in \mathbb{C}$, suitably translated so that its axis is the line $\left\{x_{1}=x_{2}=0\right\}$ and its horizontal level line at height $c$ is $\left\{\left(r e^{i(c+\pi / 2)}, c\right): r \in \mathbb{R}\right\}$. The proof of Theorem 3 will be complete if we show the following:

Claim 5. For any $\varepsilon>0$, there exists $R_{1} \geq R$ depending only on $\varepsilon$ such that $X\left(D\left(\infty, R_{1}\right)\right)$ is contained in a regular neighborhood of $H$ of radius $\varepsilon$.

In order to prove this Claim, it will be useful to introduce the following notation: given $\varepsilon>0$ and $c \in \mathbb{R}$, we denote by $\mathcal{B}(c, \varepsilon) \subset \mathbb{C}$ the circular sector centered at the origin, with amplitude $2 \varepsilon$ and bisected by the halfline $\left\{r e^{i(c+\pi / 2)}: r>0\right\}$, i.e.

$$
\mathcal{B}(c, \varepsilon)=\left\{r e^{i \theta}: r>0, c+\frac{\pi}{2}-\varepsilon \leq \theta \leq c+\frac{\pi}{2}+\varepsilon\right\} .
$$

Identifying $\mathbb{C}$ as usual with the plane $\left\{x_{3}=0\right\}$, we can consider the affine sum $\left(x_{1}, x_{2}, x_{3}\right)+\mathcal{B}(c, \varepsilon)$, which is contained in the horizontal plane at height $x_{3}$. 
Proof of Claim 5. The proof follows from a combination of the following two statements:

Statement 1 . Given $\varepsilon>0$, there exists $c_{0}>0$ depending only on $\varepsilon$ such that for any height $c$ with $|c| \geq c_{0}$ and any $v_{1} \in \mathbb{R}$, the horizontal level curve $X\left(\gamma_{c}\right)$ at this height is entirely contained in $X\left(\gamma_{c}\left(v_{1}\right)\right)+[\mathcal{B}(c, \varepsilon) \cup \mathcal{B}(c+\pi, \varepsilon)]$.

Statement 2. Given $\varepsilon>0$, there exists $v_{0}>0$ depending only on $\varepsilon$ such that for any height $c$ with $|c| \leq c_{0}$ (this is the $c_{0}$ of Statement 1), the curves $\left.X\left(\gamma_{c}\right)\right|_{\left\{v \geq v_{0}\right\}},\left.X\left(\gamma_{c}\right)\right|_{\left\{v \leq-v_{0}\right\}}$ are contained in $(0,0, c)+[\mathcal{B}(c, \varepsilon) \cup \mathcal{B}(c+\pi, \varepsilon)]$.

Assuming these two statements (which will be proved later), let us prove Claim 5 . Fix $\varepsilon>0$ and apply Statement 1 and 2 with $\varepsilon / 2$, thus we find $c_{0}, v_{0}>0$ depending only on $\varepsilon$ such that these statements hold for $\varepsilon / 2$. On the other hand, the quasivertical axes $\Gamma_{1}, \Gamma_{2}$ can be parametrized by the height $c=\operatorname{Real}(z)$ for $c$ large. As $X\left(\Gamma_{1}\right)$ (resp. $X\left(\Gamma_{2}\right)$ ) is asymptotic to the halfline $\left\{x_{1}=x_{2}=0, x_{3}<0\right\}$ (resp. $\left\{x_{1}=x_{2}=0, x_{3}>0\right\}$ ), the definition of quasivertical axis implies that we can enlarge $c_{0}$ depending only on $\varepsilon$ so that $\left|\left(x_{1}+i x_{2}\right)\left(\Gamma_{j}(c)\right)\right|<\frac{\varepsilon}{2}$ whenever $|c| \geq c_{0}$.

Given any point $X\left(\gamma_{c}(v)\right)$ with $|c| \geq c_{0}$, consider the intersection $X\left(\gamma_{c}\left(v_{j}^{c}\right)\right)$ of $X\left(\gamma_{c}\right)$ with the quasivertical axis $X\left(\Gamma_{j}\right)$. Statement 1 insures that $X\left(\gamma_{c}(v)\right)$ belongs to $X\left(\gamma_{c}\left(v_{j}^{c}\right)\right)+[\mathcal{B}(c, \varepsilon / 2) \cup \mathcal{B}(c+\pi, \varepsilon / 2)]$, thus there exists a point $\left(y_{1}, y_{2}, c\right) \in$ $(0,0, c)+[\mathcal{B}(c, \varepsilon / 2) \cup \mathcal{B}(c+\pi, \varepsilon / 2)]$ such that the distance from $X\left(\gamma_{c}(v)\right)$ to $\left(y_{1}, y_{2}, c\right)$ is less than or equal to $\varepsilon / 2$. If we write this new point $\left(y_{1}, y_{2}, c\right)$ in polar coordinates in $\left\{x_{3}=c\right\}$ as $r e^{i(c+\pi / 2+\delta)}$ with $|\delta| \leq \varepsilon / 2$, then there exists a point $P \in H$ at height $c+\delta$, on the same vertical line as $\left(y_{1}, y_{2}, c\right)$ and at distance $|\delta|$ from this last one. As a conclusion, the distance from $X\left(\gamma_{c}(v)\right)$ to $P$ is less than or equal to $\varepsilon$.

Now consider a point $X\left(\gamma_{c}(v)\right)$ with $|c| \leq c_{0}$ and $|v| \geq v_{0}$. Statement 2 implies that $X\left(\gamma_{c}(v)\right)$ itself belongs to $(0,0, c)+[\mathcal{B}(c, \varepsilon / 2) \cup \mathcal{B}(c+\pi, \varepsilon / 2)]$ and reasoning as before we find a point $P \in H$ at most at distance $\varepsilon / 2$ from $X\left(\gamma_{c}(v)\right)$, which finishes the proof of Claim 5 .

We now prove Statement 1. Fix $\varepsilon>0$ and $v_{1} \in \mathbb{R}$. It suffices to check that there exists $c_{0}>0$ depending only on $\varepsilon$ such that for $|c| \geq c_{0}$, the following two inequalities hold simultaneously for all $v$ :

$$
\begin{aligned}
& h_{c}^{+}(v):=\left\langle\left(X \circ \gamma_{c}\right)(v)-\left(X \circ \gamma_{c}\right)\left(v_{1}\right), e^{i(c+\varepsilon)}\right\rangle \geq 0, \\
& h_{c}^{-}(v):=\left\langle\left(X \circ \gamma_{c}\right)(v)-\left(X \circ \gamma_{c}\right)\left(v_{1}\right), e^{i(c-\varepsilon)}\right\rangle \leq 0 .
\end{aligned}
$$

The derivative of $h_{c}^{+}$is given by

$$
\begin{aligned}
\left(h_{c}^{+}\right)^{\prime}(v) & =\left\langle\frac{d X\left(\gamma_{c}\right)}{d v}, e^{i(c+\varepsilon)}\right\rangle=\left\langle\frac{\lambda}{|g|} i g, e^{i(c+\varepsilon)}\right\rangle=-\frac{\lambda}{|g|} \operatorname{Imag}\left(g e^{-i(c+\varepsilon)}\right) \\
& =-\lambda \sin \left(\operatorname{Imag}\left(f\left(\gamma_{c}\right)\right)-\varepsilon\right)
\end{aligned}
$$

where we have used that the derivative of $X\left(\gamma_{c}\right)$ with respect to its arc length is $\frac{d X\left(\gamma_{c}\right)}{d s}=i \frac{g}{|g|}$, that $d s=\lambda d v$ and that $\left(g \circ \gamma_{c}\right)(v)=e^{-v+i c+f\left(\gamma_{c}(v)\right)}$. Using again (19) we conclude that there exists $c_{0}>0$ depending only on $\varepsilon$ ( $c_{0}$ can be supposed strictly greater than $R$ ) such that if $|c| \geq c_{0}$, then $\sin \left(\operatorname{Imag}\left(f\left(\gamma_{c}\right)\right)-\varepsilon\right)<-\varepsilon$ along the whole domain of $\gamma_{c}$, hence for all $v \in \mathbb{R}$ it follows that

$$
h_{c}^{+}(v)=-\int_{v_{1}}^{v} \lambda \sin \left(\operatorname{Imag}\left(f\left(\gamma_{c}\right)\right)-\varepsilon\right) d v>\varepsilon \int_{v_{1}}^{v} \lambda d v \geq 0,
$$


as we desired. A similar argument holds for $h_{c}^{-}(v)$, taking into account that $\left(h_{c}^{-}\right)^{\prime}(v)=-\lambda \sin \left(\operatorname{Imag}\left(f\left(\gamma_{c}\right)\right)+\varepsilon\right)$, and Statement 1 follows (in fact, we have obtained that if $|c| \geq c_{0}$ and $v \neq v_{1}$, then $h_{c}^{+}(v)>0$ and $h_{c}^{-}(v)<0$, thereby proving that $\left(X \circ \gamma_{c}\right)(v)$ belongs to the interior of $\left.\left(X \circ \gamma_{c}\right)\left(v_{1}\right)+[\mathcal{B}(c, \varepsilon) \cup \mathcal{B}(c+\pi, \varepsilon)]\right)$.

The proof of Statement 2 is similar to the last one, by suitably changing the functions $h_{c}^{+}, h_{c}^{-}$. Given $\varepsilon>0$, we have found $c_{0}(\varepsilon)>0$ such that Statement 1 holds. Given $c$ with $|c| \leq c_{0}$ and $v$ in the domain of $\gamma_{c}$, we define

$$
H_{c}^{+}(v):=\left\langle\left(X \circ \gamma_{c}\right)(v)-c e_{3}, e^{i(c+\varepsilon)}\right\rangle, \quad H_{c}^{-}(v):=\left\langle\left(X \circ \Gamma_{c}\right)(v)-c e_{3}, e^{i(c-\varepsilon)}\right\rangle,
$$

where $e_{3}=(0,0,1)$ (note that the $c e_{3}$ is the point in the axis of the Helicoid $H$ at height $c$ ). It suffices to show that there exists $v_{0}>0$ depending only on $\varepsilon$ such that for $|c| \leq c_{0}$ and $|v| \geq v_{0}$, the inequalities $H_{c}^{+}(v) \geq 0, H_{c}^{-}(v) \leq 0$ hold simultaneously. As before,

$$
\begin{aligned}
H_{c}^{+}(v) & =H_{c}^{+}\left(v_{1}\right)+\int_{v_{1}}^{v}\left(H_{c}^{+}\right)^{\prime}(v) d v \\
& =H_{c}^{+}\left(v_{1}\right)-\int_{v_{1}}^{v} \lambda \sin \left(\operatorname{Imag}\left(f\left(\gamma_{c}\right)\right)-\varepsilon\right) d v,
\end{aligned}
$$

for any $v_{1}, v$ in the domain of $\gamma_{c}$ with $|v| \geq v_{1}$. Again, estimate (19) insures that there exists $v_{1}>0$ depending only on $\varepsilon$ such that for all $c \in \mathbb{R}$ and $v$ with $|v| \geq v_{1}$ it holds that $\sin \left(\operatorname{Imag}\left(f\left(\gamma_{c}\right)\right)-\varepsilon\right)<-\varepsilon$. Combining this inequality with (21) we have $H_{c}^{+}(v)>H_{c}^{+}\left(v_{1}\right)+\varepsilon \int_{v_{1}}^{v} \lambda d v$, and as $\lambda=\frac{1}{2}\left(|g|+|g|^{-1}\right) \geq \frac{1}{2}$ we deduce that $H_{c}^{+}(v)>H_{c}^{+}\left(v_{1}\right)+\frac{\varepsilon}{2}\left|v-v_{1}\right|$. As the function $c \mapsto-H_{c}^{+}\left(v_{1}\right)$ is continuous, it is bounded by above in the compact set $\left\{|c| \leq c_{0}\right\}$ by a positive constant $C_{0}$ depending only on $\varepsilon$, thus the inequality $H_{c}^{+}(v)>0$ holds for all $c$ with $|c| \leq c_{0}$ and for all $v$ with $|v| \geq v_{0}=v_{1}+\frac{2 C_{0}}{\varepsilon}$, which again only depends on $\varepsilon$. A similar argument holds for $H_{c}^{-}$, and Statement 2 is also proved.

\section{The Folded helicoidal END, ERRATUm to [9]}

In $[9$ the third author proved that if a properly embedded minimal end of finite type satisfies $\eta=d z$, then $g$ is of the form $g(z)=e^{i z+f(z)}$ with $f$ vanishing at infinity. One case was sadly overlooked, namely $g(z)=z^{m} e^{i z+f(z)}$ with $m= \pm 1$ and $\eta=d z$, which was eliminated by showing that such complex data cannot kill the period. It turns out such an end (which will be called a folded helicoidal end; see its geometric description later on) can exist, although it is never embedded. As a consequence, this novelty does not affect to our main Theorem 1.

We first give an example of such an end; indeed, unlike the Helicoid, the existence itself is not obvious. We need to solve the period problem around infinity. Take $a>0$ and

$$
g(z)=e^{i z} \frac{z^{2}-a^{2}}{z}, \quad \eta=d z .
$$

Then outside the disk of radius $a, g$ has the desired behaviour. Killing the period is obvious for the third coordinate, and for $\xi=x_{1}+i x_{2}$ it forces the amount to be zero in the following expression:

$$
\int \overline{g^{-1} d z}-\int g d z
$$

where the integrals run along some big circle centered at the origin. Now 


$$
\begin{aligned}
\int g d z & =\int e^{i z}\left(z-\frac{a^{2}}{z}\right) d z=-2 \pi i a^{2} \int g^{-1} d z \\
& =\int e^{-i z} \frac{z}{(z-a)(z+a)} d z=\frac{1}{2} \int e^{-i z}\left(\frac{1}{z-a}+\frac{1}{z+a}\right) d z=2 \pi i \cos a .
\end{aligned}
$$

Thus to kill the period we need $a^{2}=\cos a$; this equation has a solution approximately equal to 0.8241323123 . Notice that the flux does not vanish; the condition for that would be for both integrals $\int g d z$ and $\int g^{-1} d z$ to vanish simultaneously. On the other hand, the antiholomorphic transformation $T(z)=-\bar{z}$ preserves the metric, so it induces a symmetry of the surface. The fixed points set of this transformation consists of the two components $\gamma^{+}=[1,+\infty) i, \gamma^{-}=(-\infty,-1] i$ of the imaginary axis in the exterior of the disk of radius one (as $a \sim 0.8$ solves the period, we set the parameter domain to be $\{|z| \geq 1\}$ ), which are applied on the level set at height zero. Taking the origin of integration for the Weierstrass data at $z=i$, it follows that this antiholomorphic transformation induces a 180-degree rotation of the surface around the $x_{1}$-axis, which is contained in the surface. In particular, both $\gamma^{+}$and $\gamma^{-}$are applied by the immersion $X$ into the $x_{1}$-axis. Using equation (4) one can check that the velocity vector of both applied curves has the same constant value, hence they point to the same direction. As $\gamma^{+}, \gamma^{-}$are divergent curves and the induced metric is complete, the lengths of $X\left(\gamma^{+}\right), X\left(\gamma^{-}\right)$are infinite, thus they both are halflines contained in the same straightline and pointing to the same direction, which insures that they intersect each other and thus, the example is not embedded. Clearly, other similar examples of isolated ends can be found, but it remains an open question whether killing both period and flux is possible.

We now prove that nonembeddedness holds for any folded helicoidal end, not only for the example above. First, note that the case $m=-1$ can be reduced to $m=1$ by exchanging the orientation of the surface (i.e. write $(-1 / \bar{g}, \bar{\eta})$ instead of $(g, \eta))$. Hence let $g(z)=e^{i z}\left(z+i a+\mathcal{O}\left(z^{-1}\right)\right)$ be the Gauss map defined on $\mathbb{C}$ minus a disk, for some constant $a$, the $\mathcal{O}\left(z^{-1}\right)$ term denoting a power series in $z^{-1}$. We can suppose that $a$ is real; otherwise, picking a new variable $z-\operatorname{Im}(a)$ only rotates the surface, and gives the appropriate Gauss map. The curve at height $c$ (which is diffeomorphic to $\mathbb{R}$ or to $(-\infty,-1] \cup[1,+\infty))$ is parametrized by $\gamma_{c}(v)=c+i v$ and its horizontal projection satisfies

$$
\begin{aligned}
& \frac{d X\left(\gamma_{c}\right)}{d v}=\frac{-i}{2}\left(g+\bar{g}^{-1}\right) \\
& \quad=\frac{e^{i c}}{2}\left[v e^{-v}\left(1+\frac{a-i c}{v}+\mathcal{O}\left(v^{-2}\right)\right)+\frac{e^{v}}{v}\left(1-\frac{a+i c}{v}+\mathcal{O}\left(v^{-2}\right)\right)\right] .
\end{aligned}
$$

Proposition 2 (or direct computation of $\left\|\frac{d X\left(\gamma_{c}\right)}{d v}\right\|^{-1} \cdot \frac{d X\left(\gamma_{c}\right)}{d v}$ in (22) ) assures that for all $c \in \mathbb{R}$ the horizontal level set has two divergent branches whose arguments both converge to the argument of the same halfline $L_{c}$ starting from the origin in $\left\{x_{3}=c\right\}$ and directed by $e^{i c}$, and that $L_{c}$ rotates linearly as the height $c$ increases. In particular, both branches at height $c$ can be eventually expressed as sublinear graphs over $L_{c}$. More precisely, a branch is graphical over $L_{c}$ as soon as

$$
\begin{aligned}
\left\langle\frac{d X\left(\gamma_{c}\right)}{d v}, e^{i c}\right\rangle & =\operatorname{Real}\left[\frac{d X\left(\gamma_{c}\right)}{d v} e^{-i c}\right] \\
& =\frac{1}{2}\left[e^{-v}\left(v+a+\mathcal{O}\left(v^{-1}\right)\right)+\frac{e^{v}}{v^{2}}\left(v-a+\mathcal{O}\left(v^{-1}\right)\right)\right]
\end{aligned}
$$


has constant sign and keeps it. On the other hand, similar arguments as in Section[5 prove that there exist $c_{0}, v_{0}>0$ such that whenever $|c|>c_{0}$, the level set at height $c$ is connected and embedded (because it has absolute total curvature strictly less than $\pi$ ), while if $|c| \leq c_{0}$, the level curves $\left.X\left(\gamma_{c}\right)\right|_{\left\{v \geq v_{0}\right\}}$ and $\left.X\left(\gamma_{c}\right)\right|_{\left\{v \leq-v_{0}\right\}}$ are separately embedded, thus nonembeddedness must come from an intersection between $\left.X\left(\gamma_{c}\right)\right|_{\left\{v \geq v_{0}\right\}}$ and $\left.X\left(\gamma_{c}\right)\right|_{\left\{v \leq-v_{0}\right\}}$ for intermediate heights, as occurs in the example above.

Coming back to (23), as the terms $\mathcal{O}\left(v^{-1}\right)$ in that expression depend continuously on $c$ (they are series in negative powers of $c+i v$ ), we deduce that there exists $v_{0}>0$ such that for any height $c$ with $|c| \leq c_{0}$, it holds that $\left.X\left(\gamma_{c}\right)\right|_{\left\{v \geq v_{0}\right\}}$ and $\left.X\left(\gamma_{c}\right)\right|_{\left\{v \leq-v_{0}\right\}}$ are simultaneously graphical over $L_{c}$. Thus these graphs are controlled by the functions $Q_{c}(v)=\left\langle X\left(\gamma_{c}\right), e^{i c}\right\rangle, T_{c}(v)=\left\langle X\left(\gamma_{c}\right), e^{i\left(c+\frac{\pi}{2}\right)}\right\rangle$. Moreover, we can suitably enlarge $v_{0}$ in order to consider the continuous function $V_{c}:\left[v_{0},+\infty\right) \longrightarrow\left(-\infty,-v_{0}\right]$ that satisfies $Q_{c}\left(V_{c}(v)\right)=Q_{c}(v)$. Thus, to insure the existence of self-intersections of the surface it suffices to find a zero of the function $\delta_{c}(v)=T_{c}(v)-T_{c}(V(v))$. With this aim, note that

$$
\frac{d T_{c}}{d v}=\operatorname{Imag}\left[\frac{d X\left(\gamma_{c}\right)}{d v} e^{-i c}\right]=-\frac{c}{2}\left(e^{-v}+\frac{e^{v}}{v^{2}}\right)+e^{-v} \mathcal{O}\left(v^{-1}\right)+e^{v} \mathcal{O}\left(v^{-3}\right) .
$$

Thus there is a clear change in behavior at height zero which we sum up as follows:

1. Except at maybe one height $(c=0), \frac{d T_{c}}{d v}$ tends to $\pm \infty$ as $|t| \rightarrow \infty$,

2. When $c<0$ we have $\lim _{v \rightarrow \pm \infty} \frac{d T_{c}}{d v}=+\infty$ (thus $\lim _{v \rightarrow+\infty} \delta_{c}(v)=+\infty$ ) while when $c>0$ is $\lim _{v \rightarrow \pm \infty} \frac{d T_{c}}{d v}=-\infty$ (and $\left.\lim _{v \rightarrow+\infty} \delta_{c}(v)=-\infty\right)$.

We finally show that there exist self-intersections. Reasoning by contradiction, suppose that the end is embedded. As it is complete and its Gaussian curvature is bounded (use the estimate in the proof of Claim 3), the Regular Neighborhood Theorem in [6] implies that the end must have an embedded regular neighborhood of fixed (positive) radius; see also the proof of Claim 3 above. In particular, the two branches at height zero are at distance greater than some positive constant, thus $\delta_{0}$ stays away from zero. So we may safely assume that at height zero, $\delta_{0}$ is - for instance - greater than some positive constant. Now take $c>0$ small enough so that the level curve at height $c$ comes arbitrarily close to the one at level 0 , at least on a compact set; so $\delta_{c}$ will be arbitrarily close to $\delta_{0}$ on any compact interval, in particular, we may find $\delta_{c}(u)>0$ for $u$ as large as we want. Since $\delta_{c}(v)$ tends to $-\infty$ as $v \rightarrow+\infty$, we deduce that $\delta_{c}$ has to vanish for some $u^{\prime}>u$, i.e. the two branches intersect.

To finish this section, we prove that a folded helicoidal end can never be part of a complete (immersed) minimal surface of finite type with only one end. Let $M=\bar{M}-\{\infty\}$ be such a surface, $\bar{M}$ being a compact surface of genus $\gamma$. As $\eta$ is holomorphic on $M$ with a double pole at $\infty$, it has exactly $2 \gamma$ zeros on $M$ counted with multiplicity. Let $p_{1}, \ldots, p_{r}$ be those zeros and $k_{1}, \ldots, k_{r}$ be their multiplicities. The metric being $\frac{1}{2}\left(|g|+|g|^{-1}\right)|\eta|, g$ must have a zero or a pole of multiplicity $k_{i}$ at each $p_{i}$. That forces $d g / g$ to have a simple pole at $p_{i}$ with residue $\pm k_{i}$. These are the only zeros or poles of $g$ hence the only poles of $d g / g$ on $M$. Since $d g / g$ is meromorphic on $\bar{M}$, the sum of its residues vanishes. But the sum of the residues on $M$ is even:

$$
\pm k_{1} \pm k_{2} \pm \cdots \pm k_{r} \equiv \sum k_{i} \bmod 2
$$


and $\sum k_{i}=2 \gamma$. This contradicts that the residue of $d g / g$ at infinity is one, as can be checked by direct computation.

\section{Applications}

We now prove Theorems 1 and 4 stated in the Introduction.

Proof of Theorem 1. As $M$ is has finite type and only one end, it must be conformally $\bar{M}-\{p\}$, where $\bar{M}$ is a compact Riemann surface and $p \in \bar{M}$ corresponds to the end of $M$. As any closed curve $\Gamma$ around $p$ bounds a compact domain in $M$, the Divergence Theorem implies that the flux of $M$ along $\Gamma$ is zero. Now we can apply Theorem 2, concluding that up to a homothety, the end of $M$ can be conformally parametrized in $\{|z| \geq R\}$ by $g(z)=e^{i z+f(z)}, \eta=d z$, where $f$ is a holomorphic function on $\{|z| \geq R\} \cup\{\infty\}$ with $f(\infty)=0$. In particular, $\eta$ has a double pole without residue at the puncture, and as $d g / g=\left(i+f^{\prime}(z)\right) d z$, the same holds for $d g / g$. Finally, Theorem 3 implies that there exists a Helicoid $H$ such that for any regular neighborhood $H_{\varepsilon}$ of $H$, there exists a representative of the end of $M$ which lies inside $H_{\varepsilon}$, which completes the proof.

Proof of Theorem 母 Let $M \subset \mathbb{R}^{3}$ be a nonplanar simply connected CEMS of finite type. Thus, $M$ is conformally $\mathbb{C}$. By Theorem $1 \eta$ is a meromorphic differential on a sphere with just one pole whose order is two, so we can write globally $\eta=d z$. As a consequence, $M$ has no points with vertical normal vector, or in other words, $g(z)=e^{H(z)}$ for an entire function $H$. Using Theorem 2, $H$ must be a polynomial of degree one of the type $H(z)=a_{0}+a_{1} z$, with $a_{1}$ purely imaginary, and the theorem is proved.

\section{APPENDIX}

Lemma 3. Let $\xi(s), s \in \mathbb{R}^{+}$be a planar curve with velocity vector $\frac{d \xi}{d s}=r(s) e^{i \varphi(s)}$, where $r(s), \varphi(s)$ are smooth real valued functions of the type

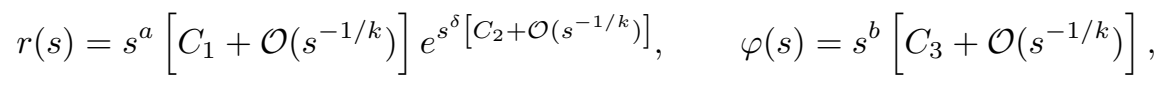

with $a, b, \delta \in \mathbb{R}, k$ is a positive integer, $b, C_{1}, C_{2}>0, C_{3} \neq 0$ and the remainder terms $\mathcal{O}\left(s^{-1 / k}\right)$ are uniformly converging series in negative powers of $s$. For any $\alpha \in[0,2 \pi)$, define $x_{\alpha}(s)=\left\langle\xi(s), e^{i(\alpha+\pi / 2)}\right\rangle, s \in \mathbb{R}^{+}$. Then, $x_{\alpha}$ has an infinite countable number of critical points, passing consecutively (for s large enough) from local maxima to local minima and vice versa. Moreover, if we denote by $\left\{s_{2 n}\right\}_{n}$ (resp. $\left\{s_{2 n-1}\right\}_{n}$ ) the sequence of consecutive local maxima (resp. local minima) of $x_{\alpha}$, then $x_{\alpha}\left(s_{n}\right)-x_{\alpha}\left(s_{n-1}\right)$ changes sign as $n$ increases, and

i) If $\delta>0$ or if $\delta=0$ and $a+1>b$, then $x_{\alpha}\left(s_{2 n}\right)$ increases to $+\infty$ and $x_{\alpha}\left(s_{2 n-1}\right)$ decreases to $-\infty$ as $n \rightarrow \infty$.

ii) If $\delta=0$ and $a+1=b$, then there exist $M(\alpha), m(\alpha), W \in \mathbb{R}$ (note that $W$ does not depend on $\alpha)$ such that $x_{\alpha}\left(s_{2 n}\right) \rightarrow M(\alpha), x_{\alpha}\left(s_{2 n-1}\right) \rightarrow m(\alpha)$ and $\left|x_{\alpha}\left(s_{2 n}\right)-x_{\alpha}\left(s_{2 n-1}\right)\right| \rightarrow W$ as $n \rightarrow \infty$.

iii) If $\delta=0$ and $a+1<b$, then $\left|x_{\alpha}(s)-x_{\alpha}\left(s^{\prime}\right)\right|$ goes to zero as $s>s^{\prime}$ tend to infinity.

Proof. As $\frac{d x_{\alpha}}{d s}=r(s) \sin (\varphi(s)-\alpha)$, the critical points of $x_{\alpha}$ coincide with the zeros of $\sin \left(\varphi(s)-\alpha\right.$ ). If $C_{3}>0$ (resp. $\left.C_{3}<0\right), \varphi(s)$ is monotonically increasing (resp. 
decreasing) to infinity for $s$ large enough. We will call $s=\psi(u)$ to its inverse function, which has the form

$$
\psi(u)=|u|^{1 / b}\left[C_{4}+\mathcal{O}\left(|u|^{-1 / b}\right)\right],
$$

for a positive constant $C_{4}$ that does not depend on $\alpha$ (in what follows, several positive constants independent of $\alpha$ will appear and will be called by $C_{4}, C_{5}, \ldots$; on the other hand, whenever an expression $E$ depends on $\alpha$ we will denote explicitly this dependence by $E(\alpha))$. As the proof is similar in the cases $C_{3}>0, C_{3}<0$, we will only deal with the first case, so $\psi^{\prime}$ is positive. Let $s_{n}=\psi(n \pi+\alpha)$ be the $n$-th extremum of $x_{\alpha}$. Thus,

$$
\begin{aligned}
\Delta_{n-1}:=x_{\alpha}\left(s_{n}\right)-x_{\alpha}\left(s_{n-1}\right) & =\int_{s_{n} \overline{1}^{1}}^{s_{n}} r(s) \sin (\varphi(s)-\alpha) d s \\
& =\int_{(n-1) \pi}^{n \pi} r(\psi(u+\alpha)) \psi^{\prime}(u+\alpha) \sin u d u .
\end{aligned}
$$

Analogously,

$$
\begin{aligned}
\Delta_{n} & =\int_{n \pi}^{(n+1) \pi} r(\psi(u+\alpha)) \psi^{\prime}(u+\alpha) \sin u d u \\
& =-\int_{(n-1) \pi}^{n \pi} r(\psi(u+\alpha+\pi)) \psi^{\prime}(u+\alpha+\pi) \sin u d u .
\end{aligned}
$$

As both $r, \psi^{\prime}$ are positive, we deduce that $\Delta_{n}, \Delta_{n-1}$ have opposite signs. Moreover, calling $h(u)=r(\psi(u)) \psi^{\prime}(u)$, it follows that

$$
\left|\Delta_{n}\right|-\left|\Delta_{n-1}\right|=\int_{(n-1) \pi}^{n \pi}(h(u+\alpha+\pi)-h(u+\alpha))|\sin u| d u,
$$

so the mean value theorem insures that

$$
2 \pi \inf _{I_{n}} h^{\prime}(u+\alpha) \leq\left|\Delta_{n}\right|-\left|\Delta_{n-1}\right| \leq 2 \pi \sup _{I_{n}} h^{\prime}(u+\alpha),
$$

where $I_{n}=[(n-1) \pi, n \pi]$. Also note that

$$
\begin{aligned}
\psi^{\prime}(u) & =u^{\frac{1}{b}-1}\left[C_{5}+\mathcal{O}\left(u^{\frac{-1}{b}}\right)\right], \\
h(u) & =u^{\frac{a+1}{b}-1}\left[C_{6}+\mathcal{O}\left(u^{\frac{-1}{k b}}\right)\right] e^{u^{\delta / b}\left[C_{7}+\mathcal{O}\left(u^{\frac{-1}{k b}}\right)\right] .}
\end{aligned}
$$

i) Suppose that $\delta>0$ or that $\delta=0$ and $a+1>b$. From (26) one checks that $h^{\prime}$ increases strictly to infinity, hence (24) implies that $\left|\Delta_{n}\right|-\left|\Delta_{n-1}\right| \rightarrow+\infty$ as $n \rightarrow \infty$. Therefore, the sequence of local maxima (resp. local minima) of $x_{\alpha}$ increases to $+\infty$ (resp. decreases to $-\infty$ ).

ii) If $\delta=0$ and $a+1=b$, then $h(u)=\left[C_{6}+\mathcal{O}\left(u^{\frac{-1}{k b}}\right)\right] e^{C_{7}+\mathcal{O}\left(u^{\frac{-1}{k b}}\right)}$, and $h^{\prime}(u)=$ $\mathcal{O}\left(u^{\frac{-1}{k b}-1}\right)$.

Now (24) gives

$$
|| \Delta_{n}|-| \Delta_{n-1}|| \leq C_{8}((n-1) \pi+\alpha)^{\frac{-1}{k b}-1} .
$$

Using repeatedly the last expression one concludes that for $n_{0}, n \in \mathbb{N}, n_{0}$ large,

$$
|| \Delta_{n_{0}+n}|-| \Delta_{n_{0}}|| \leq C_{8} \sum_{j=n_{0}}^{n_{0}+n+1}(j \pi+\alpha)^{\frac{-1}{k b}-1}
$$


that can be made smaller than any $\varepsilon$ by taking $n_{0}$ large enough. Therefore, $\left|\Delta_{n}\right|$ converges to a real number $W$ which cannot be zero and does not depend on $\alpha$ because

$$
\begin{aligned}
\left|\Delta_{n}\right| & =\int_{n \pi}^{(n+1) \pi} h(u+\alpha)|\sin u| d u \\
& =\int_{n \pi}^{(n+1) \pi}\left[C_{6}+\mathcal{O}\left(u^{\frac{-1}{k b}}\right)\right] e^{C_{7}+\mathcal{O}\left(u^{\frac{-1}{k b}}\right)}|\sin u| d u \longrightarrow 2 C_{6} e^{C_{7}} .
\end{aligned}
$$

As $\left|\Delta_{n}\right|$ converges to $W>0$ and $x_{\alpha}\left(s_{n}\right)-x_{\alpha}\left(s_{n-1}\right)$ changes sign as $n$ increases, both sequences of local maxima $x_{\alpha}\left(s_{2 n}\right)$ and local minima $x_{\alpha}\left(s_{2 n-1}\right)$ must converge, and $i i$ ) is proved.

iii) Finally, suppose that $\delta=0$ and $a+1<b$, thus, $h(u)=\mathcal{O}\left(u^{\frac{a+1}{b}-1}\right)$ and for $s>s^{\prime}$ large, we have

$$
x_{\alpha}(s)-x_{\alpha}\left(s^{\prime}\right)=\int_{u^{\prime}-\alpha}^{u-\alpha} h(v+\alpha) \sin v d v=\int_{u^{\prime}-\alpha}^{u-\alpha} \mathcal{O}\left(v^{\frac{a+1}{b}-1}\right) \sin v d v,
$$

where $s=\psi(u), s^{\prime}=\psi\left(u^{\prime}\right)$. As $\left|\int_{u_{0}}^{\infty} v^{\gamma} \sin v d v\right|<\infty$ for $\gamma<0$, we deduce that $x_{\alpha}(s)-x_{\alpha}\left(s^{\prime}\right)$ can be made smaller than any $\varepsilon>0$, which completes the proof.

Lemma 4. Let $\alpha \in[0,2 \pi)$ and $k, \lambda \in \mathbb{N}$.

i) If $\lambda$ is not a multiple of $k$, then there exist $l_{1}, l_{2} \in\{1, \ldots, 2 k\}$ such that $l_{1}-l_{2}$ is even and $\sin \left(\lambda \theta_{l_{1}}+\alpha\right) \sin \left(\lambda \theta_{l_{2}}+\alpha\right)<0$ (as before, $\theta_{l}$ are given by $\left.\theta_{l}=l \frac{\pi}{k}-\frac{\pi}{2 k}\right)$.

ii) If $(\lambda, \alpha) \neq\left(\frac{k}{2}, \frac{\pi}{4}+j \frac{\pi}{2}\right)$ with $j \in\{1, \ldots, 4\}$, then there exists $l_{0} \in\{1, \ldots, 2 k\}$ such that $\cos \left(\lambda \theta_{l_{0}}+\alpha\right) \neq 0$ and $\cos \left(\lambda \theta_{l_{0}-1}+\alpha\right) \neq 0$.

Proof. Although the argument is an elementary exercise by considering angles in the unit disk, we provide a sketch of the idea for the sake of completeness. In case $i)$, write $\lambda=p k+j$ with $p, j$ integers, $0<j<k$ and fix $l_{0} \in\{1, \ldots, 2 k\}$ where $\sin \left(\lambda \theta_{l_{0}}+\alpha\right) \geq 0$. We have two cases:

Suppose first that $\sin \left(\lambda \theta_{l_{0}}+\alpha\right)=0$. If $j \neq \frac{k}{2}$, take $l_{1}=l_{0}-2, l_{2}=l_{0}+2$ and the statement holds. If $j=\frac{k}{2}$, the choice $l_{1}=l_{0}-1, l_{2}=l_{0}+1$ works.

Assume now that $\sin \left(\lambda \theta_{l_{0}}+\alpha\right)>0$. If $j<\frac{k}{2}$ and $j \neq \frac{k}{4}$, then there exists an even integer $s$ such that $\frac{k}{2 j}<s<\frac{k}{j}$, and we choose $l_{1}=l_{0}$ and $l_{2}=l_{0}-s$ or $l_{2}=l_{0}+s$, depending on the possibilities $\cos \left(\lambda \theta_{l_{0}}+\alpha\right)>0$ or $\cos \left(\lambda \theta_{l_{0}}+\alpha\right) \leq 0$, respectively. If $j=\frac{k}{4}$, take $l_{1}=l_{0}$ and $l_{2}=l_{0}-2$ (resp. $\left.l_{2}=l_{0}+2, l_{2}=l_{0}+4\right)$ provided that $\cos \left(\lambda \theta_{l_{0}}+\alpha\right)$ is positive (resp. negative, zero). If $\frac{k}{2} \leq j<k$ and $\cos \left(\lambda \theta_{l_{0}}+\alpha\right) \geq 0$, then there exists a positive even integer $t$ such that $\lambda \theta_{l_{0}+t}+\alpha$ is in the open lower halfdisk, so we can take $l_{1}=l_{0}$ and $l_{2}=l_{0}+t$. Finally, if $\frac{k}{2} \leq j<k$ and $\cos \left(\lambda \theta_{l_{0}}+\alpha\right)<0$, we can choose a positive even integer $t$ such that $\lambda \theta_{l_{0}-t}+\alpha$ is in the open lower halfdisk and we finish with $l_{1}=l_{0}, l_{2}=l_{0}-t$. This finishes $i$ ) of our lemma.

Concerning ii), suppose first that $\lambda=\frac{k}{2}$. Thus, $\lambda \theta_{l}+\alpha=l \frac{\pi}{2}-\frac{\pi}{4}+\alpha$, which cannot be a zero of the cosine function because $\alpha \neq \frac{\pi}{4}+j \frac{\pi}{2}$. Finally, assume $\lambda \neq \frac{k}{2}$. Note that the difference between $\lambda \theta_{l}+\alpha$ and $\lambda \theta_{l-1}+\alpha$ is $\left.\lambda \frac{\pi}{k} \in\right] 0, \pi\left[-\left\{\frac{\pi}{2}\right\}\right.$. Reasoning by contradiction, if ii) does not hold, then we can choose $l \in\{1, \ldots, 2 k\}$ 
such that $\cos \left(\lambda \theta_{l}+\alpha\right)=0$. Therefore, neither $\cos \left(\lambda \theta_{l-1}+\alpha\right)$ nor $\cos \left(\lambda \theta_{l-2}+\alpha\right)$ vanish, a contradiction.

\section{REFERENCES}

[1] P. Collin, Topologie et courbure des surfaces minimales proprement plongees de $\mathbb{R}^{3}$, Ann. of Math. 2nd Series 145 (1997) 1-31. MR 98d:53010

[2] D. Hoffman \& H. Karcher, Complete embedded minimal surfaces of finite total curvature, in R. Osserman editor, Encyclopedia of Mathematics, volume Minimal Surfaces, pages 5-90. Springer, 1997. MR 98m:53012

[3] D. Hoffman, H. Karcher \& F. Wei, The genus one helicoid and the minimal surfaces that led to its discovery, Global Analysis and Modern Mathematics, Karen Uhlenbeck, editor, Publish or Perish Press (1993) 119-170. MR 95k:53011

[4] D. Hoffman \& J. McCuan, Embedded minimal ends asymptotic to the Helicoid, preprint.

[5] W. H. Meeks, III \& H. Rosenberg, The geometry of periodic minimal surfaces, Comm. Math. Helv. 68 (1993) 538-578.

[6] W. H. Meeks III \& H. Rosenberg, Maximum principles at infinity with applications to minimal and constant mean curvature surfaces, preprint.

[7] L. Rodríguez \& H. Rosenberg, Some remarks on complete simply connected minimal surfaces meeting the planes $x_{3}=$ constant transversally, Geom. Anal. 7 (1997), 329-342. MR 2000a:53015

[8] L. Rodríguez \& H. Rosenberg, Minimal surfaces in $\mathbb{R}^{3}$ with one end and bounded curvature, Manuscripta Math. 96 (1998) 3-7. MR 99e:53010

[9] P. Romon, On helicoidal ends of minimal surfaces, Ann. of Global Anal. and Geom. 12 (1994) 341-355. MR 95k:53012

[10] H. Rosenberg, Minimal surfaces of finite type, Bull. Soc. Math. France, 123 (1995) 351-359. MR 97a:53011

[11] H. Rosenberg \& E. Toubiana, Simply connected minimal surfaces in $\mathbb{R}^{3}$ transverse to horizontal planes, Ann. of Global Anal. and Geom. 16 (1998) 89-100. MR 99e:53011

[12] F. Xavier, Why no new complete simply-connected embedded minimal surfaces have been found since 1776, preprint.

Department of Mathematics, University of Fortaleza, 60811-341 Fortaleza, Brazil Current address: Equipe d'Analyse et de Mathematiques Appliquees, Universite de Marne-laVallee, 2 rue de la Butte Verte, 93166 Noisy-le-Grand Cedex, France

E-mail address: hauswirth@math.univ-mlv.fr

Departamento de Geometria y Topologia, Universidad de Granada, Fuentenueva s/n, 18071, Granada, Spain

E-mail address: jperez@goliat.ugr.es

Equipe D'Analyse et de Mathematiques Appliquees, Universite de Marne-la-Vallee, 2 rue de la Butte Verte, 93166 Noisy-le-Grand Cedex, France

E-mail address: romon@math.univ-mlv.fr 